\title{
Metal-to-Insulator Transition in Ultrathin Manganite Heterostructures
}

\author{
Zhaoliang Liao ${ }^{1}$ and Jiandi Zhang ${ }^{2, *}$ \\ 1 National Synchrotron Radiation Laboratory, University of Science and Technology of China, Hefei 230026, \\ Anhui, China; zliao@ustc.edu.cn \\ 2 Department of Physics and Astronomy, Louisiana State University, Baton Rouge, LA 70810, USA \\ * Correspondence: jiandiz@lsu.edu
}

Received: 24 November 2018; Accepted: 27 December 2018; Published: 3 January 2019

\begin{abstract}
Thickness-driven phase transitions have been widely observed in many correlated transition metal oxides materials. One of the important topics is the thickness-driven metal to insulator transition in half-metal $\mathrm{La}_{2 / 3} \mathrm{Sr}_{1 / 3} \mathrm{MnO}_{3}$ (LSMO) thin films, which has attracted great attention in the past few decades. In this article, we review research on the nature of the metal-to-insulator (MIT) transition in LSMO ultrathin films. We discuss in detail the proposed mechanisms, the progress made up to date, and the key issues existing in understanding the related MIT. We also discuss MIT in other correlated oxide materials as a comparison that also has some implications for understanding the origin of MIT.
\end{abstract}

Keywords: metal to insulator transition; manganite; oxide heterostructure; dead layer; LSMO

\section{Introduction}

Thanks to the fast development of advanced thin film epitaxial techniques, such as pulsed laser deposition (PLD) and molecular beam epitaxy (MBE), artificial low-dimensional materials can be fabricated with thickness that is controlled precisely down to the atomic scale. This provides unprecedented opportunities to tailor complex oxides for new functionalities by material architecture with atomic precision [1-3]. By introducing strain [4,5], interface [6,7], broken symmetry [8,9], and by reducing dimensionality [10], new matter states or novel functionalities have been realized in oxide heterostructures, which are fundamentally different from those exhibited by their corresponding bulk forms. Typical examples include the interface-induced multiferroism [11], the polar metal in geometrically designed nickelate heterostructures [12] and the polarized vortex in $\mathrm{PbTiO}_{3} / \mathrm{SrTiO}_{3}$ superlattices [13], etc. It is widely believed that novel phenomena are driven by tailoring the intimate coupling between spin, charge, orbital, and lattice. Though the underline physics is still too complicated to fully understand in transition metal oxide heterostructures, thickness variation becomes a very common and simple approach to induce emergent physical properties. Particularly crucial is when the layers enter into the ultrathin region, where the thickness is only several unit cells (u.c.) or a few nanometers. Many phase transitions, such as the metal-to-insulator transition [14-18], ferromagnetism to anti/paramagnetism [16-19], ferroelectricity to paraelectricity [20], have been found by reducing the thickness by even just one unit cell, in different kinds of ultrathin perovskite thin films. Besides the rich fundamental physics, understanding the mechanism of thickness-driven collective phase transitions is very important for the next-generation miniature electronic devices.

Among these emergent phenomena, the metal-to-insulator transition (MIT) in manganites $\mathrm{La}_{1-x} \mathrm{Sr}_{x} \mathrm{MnO}_{3}$ thin films is a highly focused topic. $\mathrm{La}_{1-x} \mathrm{Sr}_{x} \mathrm{MnO}_{3}$ exhibits perovskite structure with corner-shared $\mathrm{MnO}_{6}$ octahedra forming a 3-dimensional network (see Figure 1a). Depending on the doping level, $\mathrm{La}_{1-x} \mathrm{Sr}_{x} \mathrm{MnO}_{3}$ has different crystalline symmetry and rich electronic ground 
states, as shown in Figure 1b [21]. Near both end sides of the phase diagram $(x<0.1 \& x>0.5)$, an antiferromagnetic insulating phase is found to be the ground state, while it is a ferromagnetic metal in between states $(0.2<x<0.5)$. The most striking properties of $\mathrm{La}_{1-x} \mathrm{Sr}_{x} \mathrm{MnO}_{3}$ are above room temperature (Curie temperature) and near $100 \%$ spin polarization in $x=1 / 3 \mathrm{La}_{2 / 3} \mathrm{Sr}_{1 / 3} \mathrm{MnO}_{3}$ (LSMO) [22,23], which is also often written as the compound $\mathrm{La}_{0.7} \mathrm{Sr}_{0.3} \mathrm{MnO}_{3}$. LSMO is the most itinerant electron material, with the highest Curie temperature $\left(T_{\mathrm{C}} \sim 369 \mathrm{~K}\right)$ among all of the known manganites [22]. These unique properties make LSMO an extremely interesting material for technological applications [24,25]. Efforts to obtain high quality epitaxial LSMO films can be traced back to the 1990s, by magnetron sputtering deposition and PLD [26-30]. The PLD technique was the key technique for growing LSMO films, and bulk-like properties in LSMO thin films have been reproduced by PLD, allowing us to fabricate LSMO-based spintronics devices [27-30]. Excellent device functionalities have been demonstrated in LSMO spintronics devices $[24,25,29,30]$. Reducing the thickness of LSMO is crucial for the miniaturization of the device. However, it becomes very challenging to obtain high-quality ultrathin LSMO films, since the properties of LSMO are greatly deteriorated when the thickness is reduced down to a few unit cells [31-41]. The LSMO films undergo a metal to insulator transition with reducing thickness. Furthermore, the magnetic moment and Curie temperature were also found to decrease with reducing thickness. This layer, where the bulk functionality is destroyed, is named a "dead" layer in common jargon. It is also found that the magnetic dead layer is normally thinner than the electric dead layer [18,32,39], indicating the decoupling between ferromagnetism and metallicity. The existence of this dead layer provides disordered spins in the tunnel barrier, and hinders its application for the ultimate miniaturization of spintronics devices.

(a)

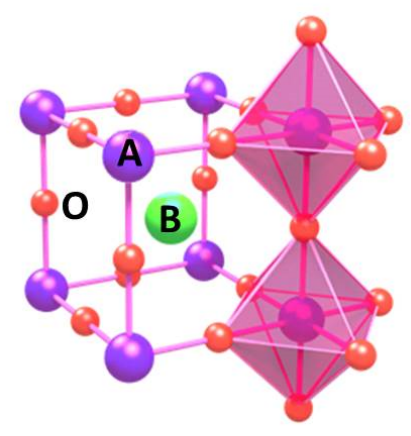

(b)

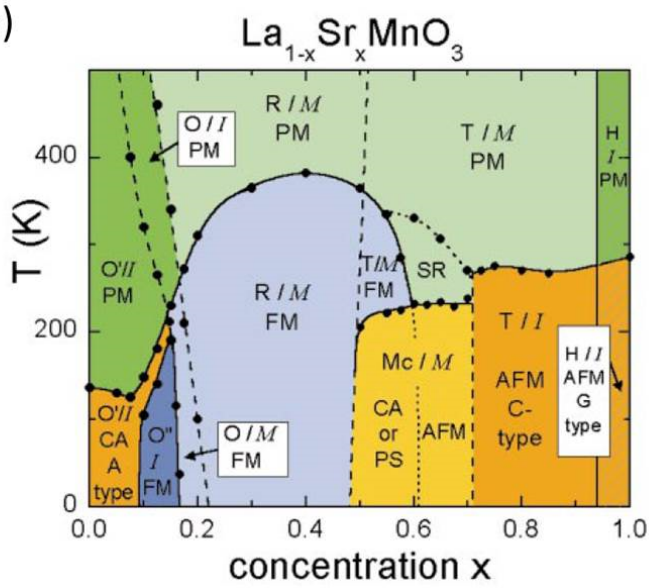

Figure 1. (a) Schematic structure model of $\mathrm{ABO}_{3}$ perovskite. (b) Phase diagram of $\mathrm{La}_{1-x} \mathrm{Sr}_{X} \mathrm{MnO}_{3}$. (Figure is adapted from Ref. [21]). The phases are denoted by capital labels: Insulator (I), Metallic (M); Jahn-Teller distorted $\left(\mathrm{O}^{\prime}\right)$, orthorhombic $(\mathrm{O})$, orbital-ordered orthorhombic $\left(\mathrm{O}^{\prime \prime}\right)$, rhombohedral $(\mathrm{R})$, tetragonal (T), monoclinic (Mc), hexagonal $(\mathrm{H})$; paramagnetic $(\mathrm{PM})$ (green); short-range order (SR), canted (CA), A-type antiferromagnetic: AFM, ferromagnetic (FM), phase-separated (PS).

It has been speculated that this dead layer exists as well in thick films at interfaces, or at surfaces [40,42-44]. This picture has been confirmed later by newly developed resonant X-ray reflectivity (RXR) [40,45] and polarized neutron reflectivity (PNR) [42-44], which are able to spatially resolve the magnetization profile at sub-nm scales. This interfacial dead layer introduces unnecessary spin-scattering in the magnetic tunneling junction, and degrades the performance of the devices. Aiming to functionalize the LSMO for practical spintronics applications, tremendous efforts have been paid to investigate the mechanism of the dead layer.

Two main questions have to be answered. First, what is the minimum thickness of the dead layer? Second, what is the mechanism behind it? These two questions are actually related to each other. The answer to the second question should be able to answer the first question. The answer to the first 
question also provides evidence to the second question. From a technological aspect, we would like to avoid the dead layer. In the aspect of fundamental research, we would like to obtain more insight into the intrinsic physics of oxide heterostructures. In this review, we try to cover these two questions by reviewing the closely related research work in the past. In Section 2, we will introduce epitaxial growth techniques. Then an overview of the research on the dead layer is given in Section 2. More detailed research on the origin of the dead layer will be discussed in Section 4. In Section 5, we also briefly describe the dead layer in other correlated perovskite oxides. A future prospective view is made at the end of this review (Section 6).

\section{Epitaxial Growth of LSMO}

The LSMO films have been generally grown by PLD. Another technique of oxide-MBE (O-MBE) has recently been used as well, for LSMO growth. In this review, we will only introduce the growth of LSMO by PLD systems. A PLD is normally made of a $\mathrm{KrF}$ excimer laser; a rotational multiple-target assembly; a gas flow nozzle; a substrate heater, and Reflection High-Energy Electron Diffraction (RHEED) (see Figure 2a). The dynamic growth process can be monitored in real time by RHEED. In order to fabricate the multilayer, a multi-target holder is usually installed. Through choosing substrates with the proper lattice mismatch, and using the optimized growth condition, a layer-by-layer growth can be obtained. Since layer-by-layer growth will generate an intensity oscillation in RHEED diffraction spots, the thickness of the film can be precisely controlled at the unit cell level. Figure $2 b$ displays a sketch of an oxide thin film on a substrate, where a further layer only starts to form when the existing surface is fully covered.

(a)

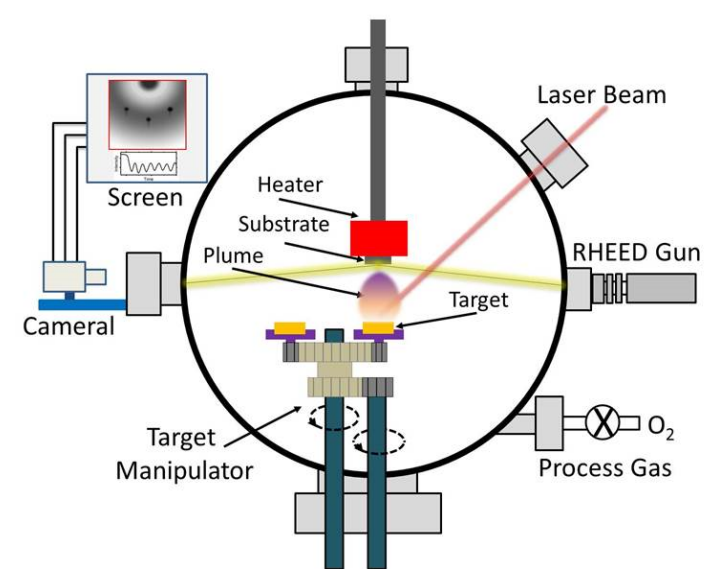

(b)

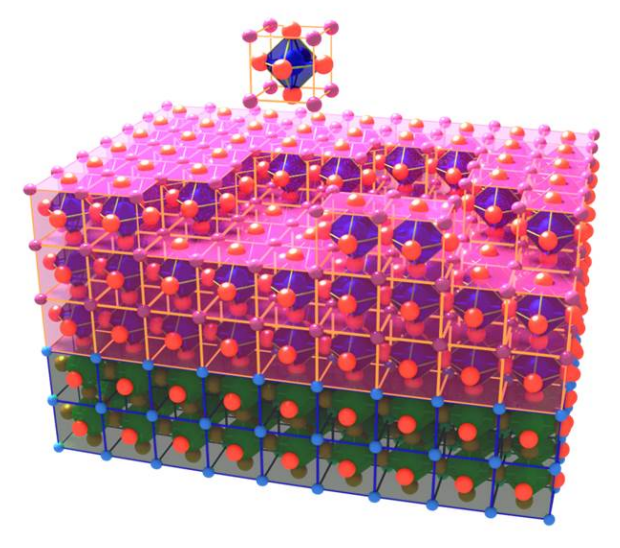

Figure 2. (a) Schematic drawing of a pulsed laser deposition setup. (b) Unit cell control of the synthesis of a specific $\mathrm{ABO}_{3}$ perovskite film (top layer) on a $\mathrm{A}^{\prime} \mathrm{B}^{\prime} \mathrm{O}_{3}$ pervoskite substrate (bottom layer).

The precise control of thickness is in detail illustrated by an example shown in Figure 3. Here, the $\mathrm{LSMO}$ films were grown on $\mathrm{TiO}_{2}$-terminated $\mathrm{SrTiO}_{3}$ (STO) (001) substrates [39]. Figure 3a,b shows the surface morphology of an as-treated STO substrate and an as-grown LSMO film, by atomic force microscopy (AFM), indicating an atomically flat surface. The atomically flat surfaces are also indicated from the 2D feature of RHEED diffraction pattern for both the STO substrate and the LSMO thin film (see the inset of Figure 3a,b). The real-time tracking of diffraction spot intensity simultaneously during the growth is shown in Figure 3c. RHEED intensity oscillation was observed for different oxygen growth partial pressure. One oscillation corresponds to the growth of one unit cell of LSMO. The observed slower growth rate in higher oxygen pressure is due to the side scattering of the plume by background gas, which reduces the amount of arriving species on substrates. The thickness can be controlled by regulating the laser ablation after a certain amount of oscillation. The atomically sharp interface between LSMO/STO was characterized by scanning transmission electron microscopy 
(STEM), as shown in Figure 3d. There is no obvious structure defect according to the STEM cross-section image. To understand the nature of the emergent properties at interface and ultrathin films, including the nature of thickness-driven MIT, it is essential to have atomic precision in both controlling thin film growth, and characterizing lattice structure and chemical composition using a technique such as high-resolution STEM.
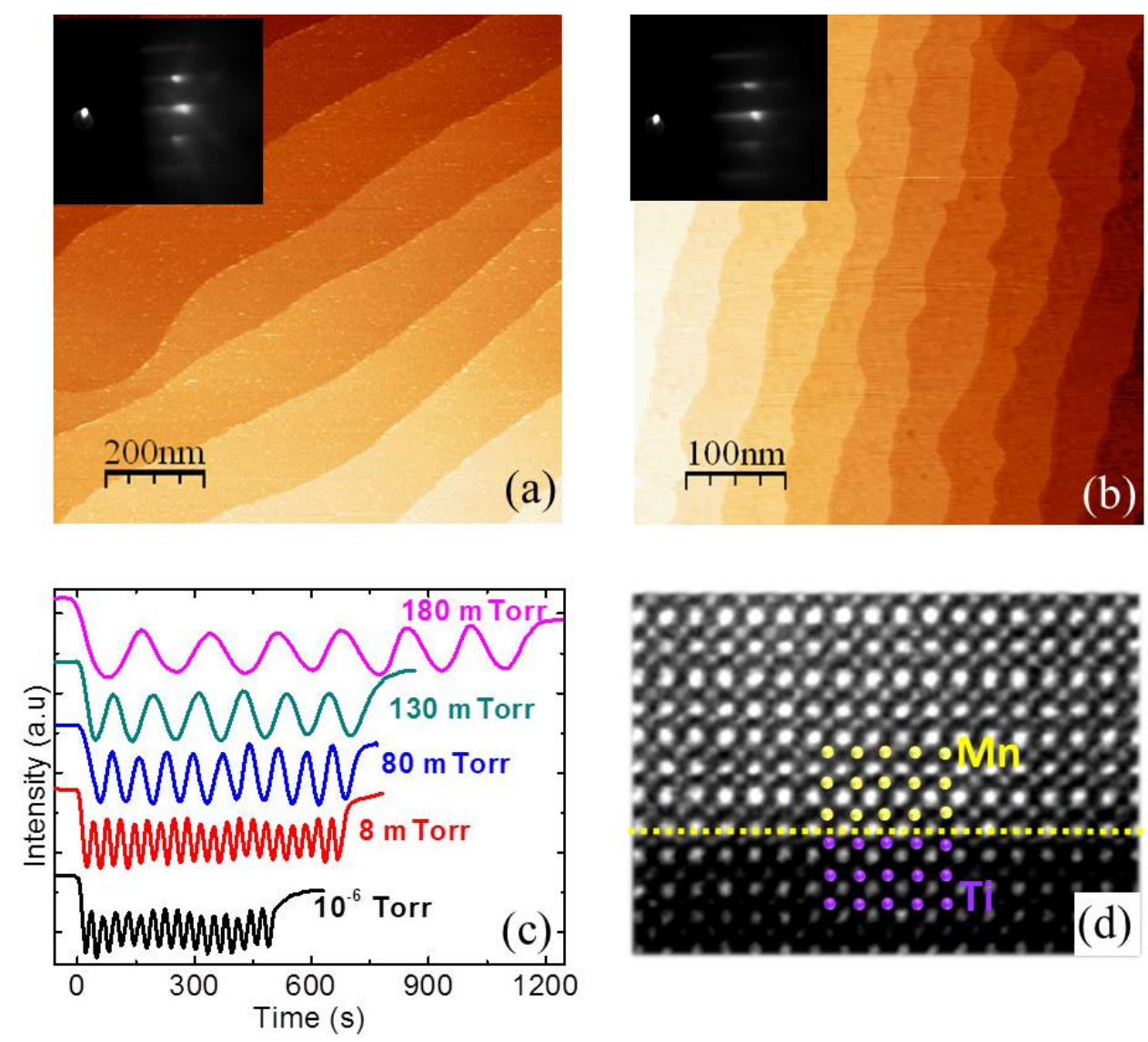

Figure 3. Surface morphology of (a) the $\mathrm{SrTiO}_{3}$ substrate and (b) 10 u.c. $\mathrm{La}_{2 / 3} \mathrm{Sr}_{1 / 3} \mathrm{MnO}_{3}$ (LSMO) thin film imaged by scanning tunneling microscopy. Inset of (a) and (b) show the corresponding Reflection High-Energy Electron Diffraction (RHEED) pattern. (c) RHEED intensity monitored during growth at different oxygen partial pressures. (d) Cross-section of a scanning transmission electron microscopy (STEM) image of the $\mathrm{LSMO} / \mathrm{STO}\left(\mathrm{TiO}_{2}\right.$-terminated $\left.\mathrm{SrTiO}_{3}\right)$. Figure is adapted from Ref. [39].

\section{The Dead Layer in LSMO}

The bulk LSMO is suggested to be half-metal, but the epitaxial LSMO films are found to gradually become insulating with reducing thickness. A thickness-driven MIT can be found, where above and below a critical thickness, the film is metallic and insulating, respectively, as shown by an example of LSMO films grown on STO substrates (Figure 4a) [39]. The insulating LSMO film is defined as the electrical dead layer. Although there are a lot of reports of the dead layer thickness from different groups, the exact thickness of the electric dead layer is not conclusive. The thicknesses of electric dead layer on different substrates reported by different group are listed in Figure $4 \mathrm{~b}$. On the same substrate, for example, STO, quite different thicknesses of dead layer, e.g., $4.0 \mathrm{~nm}$ [33], $3.1 \mathrm{~nm}$ [18], $2.7 \mathrm{~nm}$ [35], and $2.3 \mathrm{~nm}$ [39], have been reported. The reported thicknesses of the dead layer on the $\mathrm{LaAlO}_{3}(\mathrm{LAO})$ substrate even has a big gap (5 nm [31] vs $12 \mathrm{~nm}[34,39])$. There must be some extrinsic elements causing the dead layer. For example, with the presence of oxygen deficiency during growth, due to an oxidizing background that is not strong enough, gas is found to increase the dead layer thickness [39]. The growth condition, e.g., oxygen partial pressure-dependent Sr-segregation near the top surface is 
also expected to vary the actual dead layer thickness [46-49]. Interdiffusion would also play a role in deteriorating the properties, and contribute to the origin of the dead layer $[44,50]$.
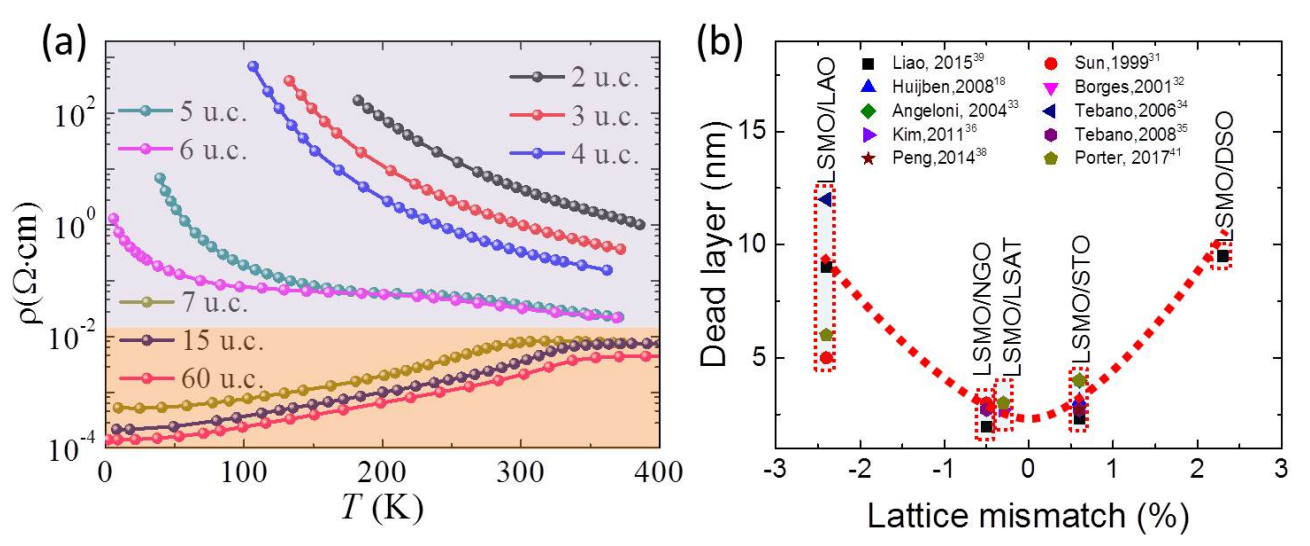

Figure 4. (a) Temperature-dependent resistivity of different thickness of LSMO thin films grown on STO (001) substrate. Figure is adapted from Ref. [39]. (b) Reported thicknesses of the dead layer of LSMO thin films grown on different substrates. $\varepsilon \equiv\left(a_{\text {Sub }}-a_{\text {LSMO }}\right) / a_{\text {LSMO }}$.

Although there is big discrepancy about the dead layer thickness reported from different research groups, a statistical tendency that dead layer thickness is smaller with smaller strain can be observed if plotting the dead layer thickness as a function of the lattice mismatches, as shown in Figure $4 \mathrm{~b}$. This fact strongly suggests an important role of lattice strain in inducing the dead layer, and a minimum dead layer thickness will be expected for a non-strained LSMO films. Unfortunately, ideal freestanding LSMO films cannot be experimentally synthesized, leaving an open question on what is the intrinsic dead layer that is driven by solely dimensionality effect.

Since the existence of the dead layer can degrade the device performance, and hinder the miniaturization of the device, it is an important research goal for material science communities to remove the dead layer. Though the underlying physics for the dead layer is still not fully understood, research in the past few decades has already revealed the important role of stoichiometry (oxygen deficiency, cation off-stoichiometry, Sr-segration, interdiffusion), surface broken symmetry-induced structure and electronic reconstruction, charge transfer, and strain in affecting the dead layer, laying a frame for engineering, and eliminating the dead layer. A thinner dead layer is expected when those factors can be mitigated or eliminated. Hereto, an ultrathin dead layer of 3 u.c. was achieved in STO-sandwiched LSMO grown on $\mathrm{NdGaO}_{3}$ (NGO) (110) substrate by combining the growth condition optimization, strain reduction, interface engineering, and surface capping [39]. It was found that the thickness of the dead layer decreased with increasing oxygen pressure [39]. By using high ozone partial pressure the as background gas during the growth, oxygen deficiency was minimized. The NGO substrate had a smaller lattice mismatch $(-0.5 \%)$ with LSMO. The STO buffer layer inserted between LSMO and NGO plays a role to reduce octahedra tilting via the octahedral coupling effect [40]. The capping layer alleviates/eliminates surface distortion, and restores the surface magnetization, as a result further reducing the dead layer thickness. These optimization leads to a record of the thinnest dead layer of only 3 u.c. (1.2 nm) [39]. As shown in Figure 5, the 4 u.c. LSMO film still exhibits very good metallic behavior, with resistivity of only $1 \mathrm{~m} \Omega \cdot \mathrm{cm}$ at $2 \mathrm{~K}$. For the 3 u.c. film, we can still observe a MIT at $80 \mathrm{~K}$, though it quickly reenters into insulating phase at $42 \mathrm{~K}$. The MIT at $80 \mathrm{~K}$ should be due to the existence of paramagnetic to ferromagnetic transition, while the low temperature reentrant insulator phase should be related to the electron localized effect, due to unavoidable disorder. This fact suggests that the magnetic dead layer should be no more than 2 u.c. Of other thickness-dependent magnetization measurements [18,40], layer-resolved magnetization [40,43,44] also suggests that the magnetic dead layer can be as small as a single unit cell. All of these results indicate that the dimensionality effect plays a minor role for the formation of the dead layer. 


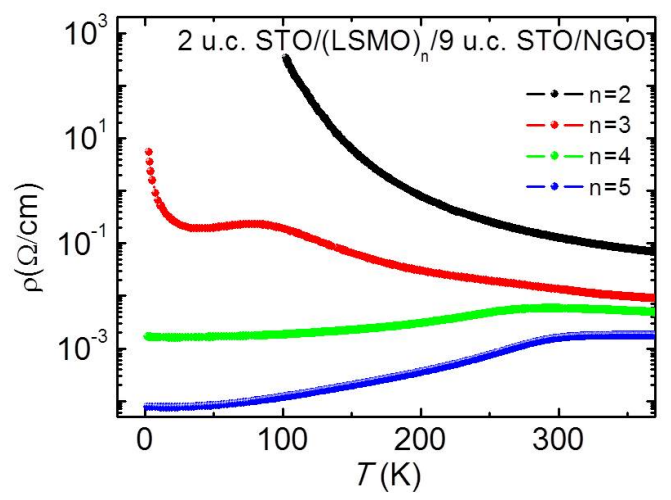

Figure 5. Temperature dependent resistivity of different thicknesses of STO-sandwiched LSMO layers grown on $\mathrm{NdGaO}_{3}$ (110) substrate. Figure is adapted from Ref. [39].

The magnetic dead layer thickness can be resolved by determining the magnetic profile along the surface normal direction. The magnetic depth profile can be obtained by RXR or PNR [40,42-45]. Figure 6 shows an example of the magnetic profile obtained by RXR [40]. The chemical profile is shown as well, for comparison. The 6 u.c. LSMO films showed a magnetic dead layer near the top surface of the LSMO layers, and suppressed magnetization near the interface (see Figure 6a). With the STO buffer layer, the interfacial magnetization was restored (see Figure $6 \mathrm{~b}$ ), which should be due to the reduced structure distortion [40]. However, the surface dead layer still existed. This suggests that the surface dead layer should be related to surface broken symmetry. However, the surface dead layer was absent when the sample was capped with STO, both for the buffered and non-buffered LSMO films (see Figure $6 c, d$ ). The stoichiometry profiles extracted from RXR for both buffered and non-buffered LSMO films verified their similarities and their sharp chemical contrasts across interfaces. RXR also revealed a stoichiometric profile from the interface to the bulk region except the top surface. The variation of the La/Sr stoichiometry near the top surface is probably important for explaining the role of the capping layer, since the capped samples have a more stoichiometric surface than the non-capped samples. The enhanced surface structural distortion, as indicated by DFT calculation (see Supplementary information in Ref. [40]) and experimentally confirmed by surface X-ray diffraction [51], should play a strong role in inducing a surface dead layer. The STO capping layer can enhance the surface magnetism by reducing the surface distortion and minimizing the surface off-stoichiometry.
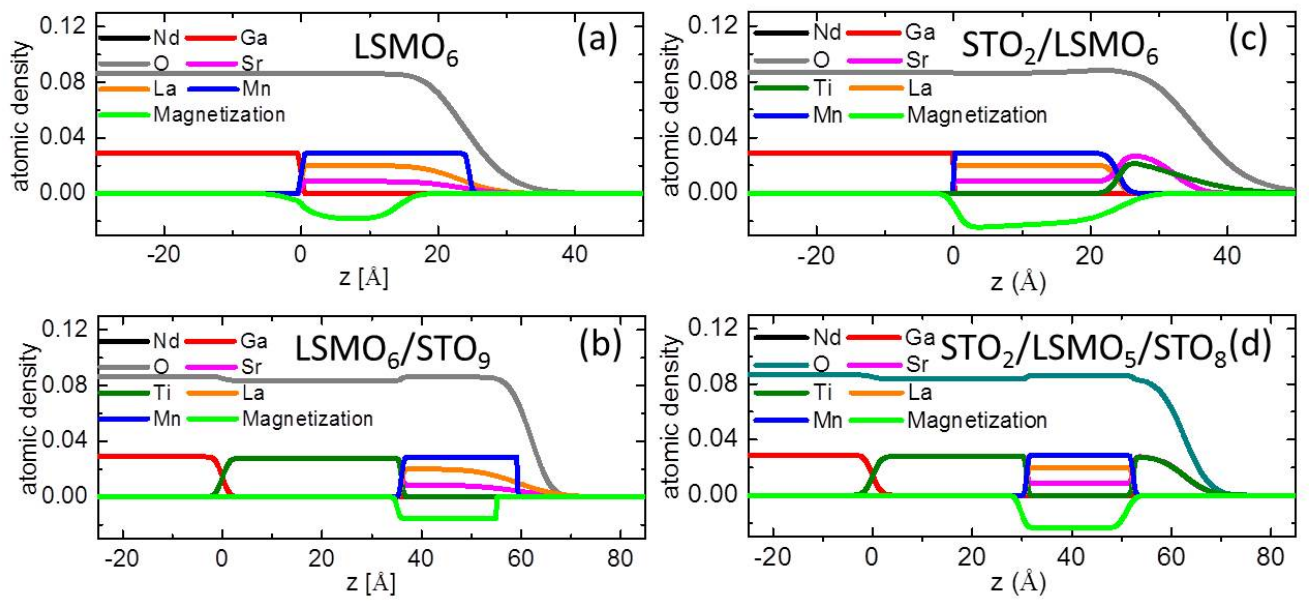

Figure 6. Stoichiometry and magnetic profiles for different LSMO films with/without STO buffer/capping layers. The depth profiles of the $\mathrm{Ga}, \mathrm{Ti}, \mathrm{Mn}, \mathrm{Nd}, \mathrm{La}, \mathrm{Sr}, \mathrm{O}$ atomic concentration, and Mn magnetization were measured at $20 \mathrm{~K}$. Reproduced with permission from Liao et al. [40]. Copyright 2017 John Wiley and Sons. 


\section{Mechanism of the MIT}

Since the existence of dead layers greatly degrade the performance of LSMO-based devices, tremendous efforts have been paid to investigate the origin of dead layers, leading to deep insight into the mechanism of the dead layer, from both experimental and theoretical points of view. A lot of detailed information on lattice distortion, charge distribution, and stoichiometry provide us many aspects of the LSMO dead layers. Several models have been proposed. The reconstruction of the Mn $e_{\mathrm{g}}$ orbital [34,35,37,52-54], reduced orbital hybridization (bandwidth) [40,47,51], the change of Mn valence [46-48,53-56] and structure imperfection (defects, disorder) $[39,44,50,57]$ are the four main proposed mechanisms in the past, which are summarized in Figure 7. Even for a single proposed mechanism, e.g., the orbital reconstruction, the origin associated with it can be quite different. The orbital reconstruction can arise from strain-induced octahedral deformation [34,52], an interface interaction favoring an out-of-plane orbital [37] or a broken symmetry modified orbital configuration [35,52]. Valence change can be driven by charge transfer due to polar mismatch $[55,56]$, or chemical reconstruction due to interdiffusion or Sr-segregation [46-49]. The past research on one hand shows the complexity and diverse variation in LSMO, and on the other hand, it indicates that the dead layer is a result of cooperative effects from contributions such as strain, off-stoichiometry, and charge variation.
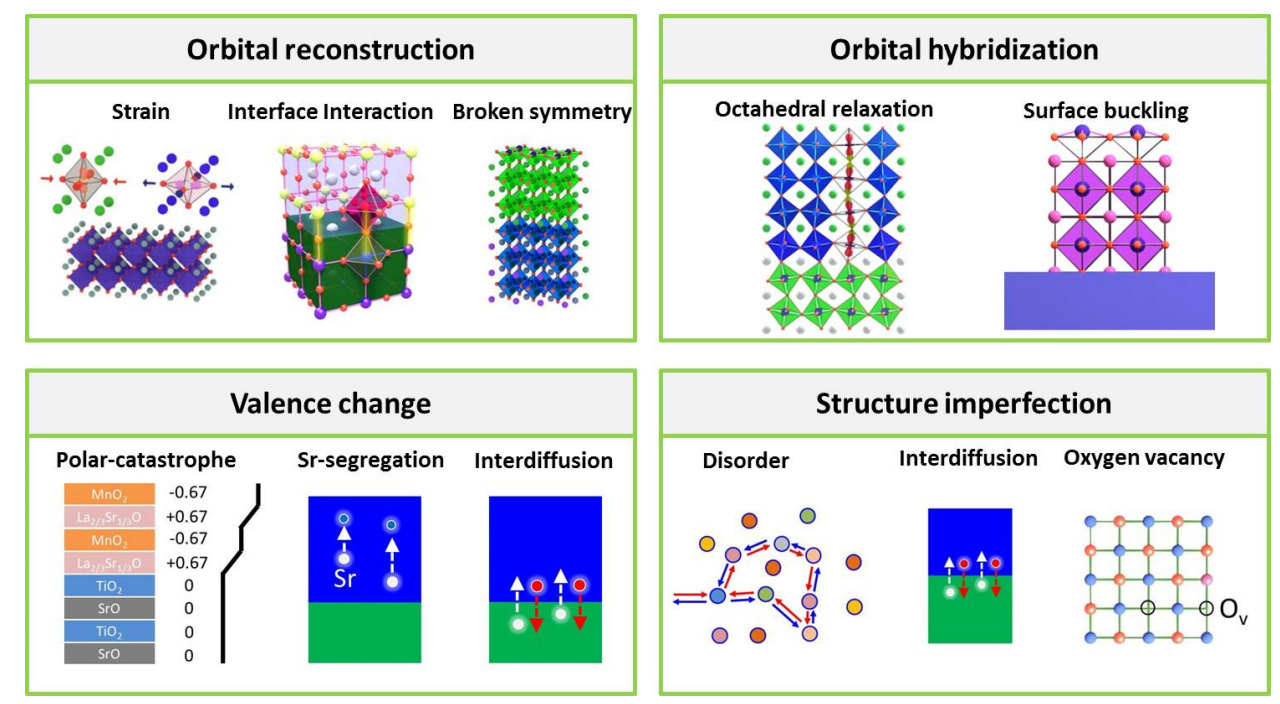

Figure 7. Proposed mechanisms for the origin of the dead layer in LSMO thin films.

\subsection{Orbital Reconstruction}

In bulk LSMO, the orbital is degenerate, and the double exchange is isotropic, leading to 3D ferromagnetic (FM) ordering. However, either $3 z^{2}-r^{2}$ or $x^{2}-y^{2}$ orbital ordering will lead to strong anisotropic transfer integral, and thus C-type AFM or A-type antiferromagnetism (AFM) (see Figure 8), respectively. For epitaxial LSMO films, a common reason to favor orbital reconstruction is the tetragonal distortion due to strain imposed by substrates. As shown in Figure 8, compressive strain favors the elongation of the octahedra, while tensile strain will flatten the octahedra, thus leading to the preference of $3 z^{2}-r^{2}$ or $x^{2}-y^{2}$ orbital occupancy, respectively [58]. No matter whether it is tensile or compressive strain, the AFM ground state is stabilized with respect to the FM, therefore driving the system to be an AFM insulator. This is true for LSMO under large degrees of strain, e.g., on LSMO/LAO

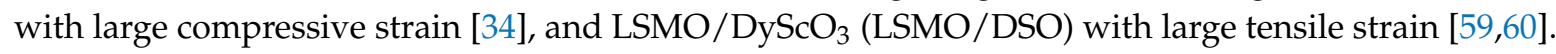
Using polarization-dependent (in-plane $\sigma$ and out-of-plane $\pi$ ) X-ray absorption (XAS), Tebano et al. revealed a $3 z^{2}-r^{2}$ orbital ordering in LSMO/LAO [34]. On the other side, when LSMO is under large tensile strain, e.g., LSMO/DSO, the $x^{2}-y^{2}$ orbital is more populated [59]. Accompanying the large orbital polarization, a very thick dead layer of about $10 \mathrm{~nm}$ is observed in LSMO/LAO and 
LSMO/DSO $[34,39,59]$. The metallic behavior in thick LSMO films on LAO or DSO starts to recover when the strain relaxes in thick films.

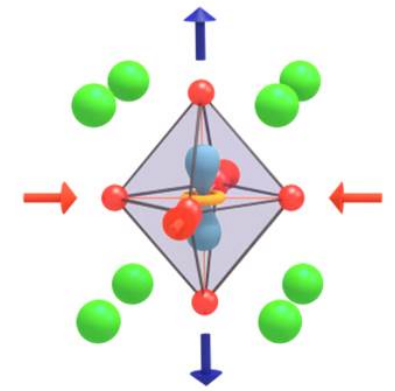

Tensile Strain

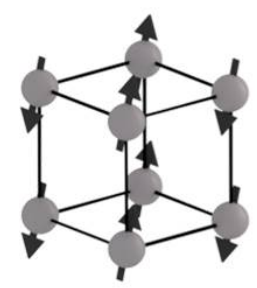

C-AFM

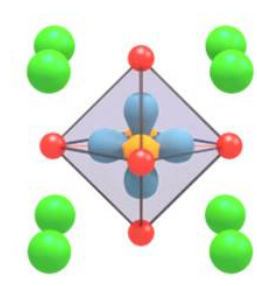

No-strain

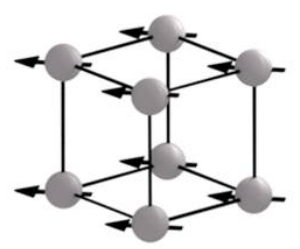

FM

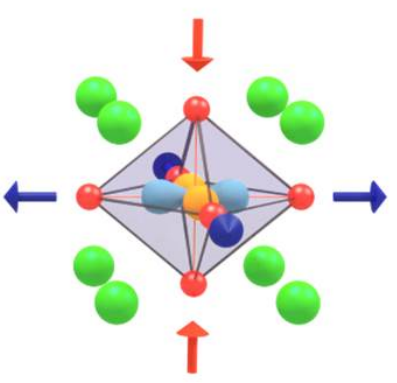

Compressive Strain

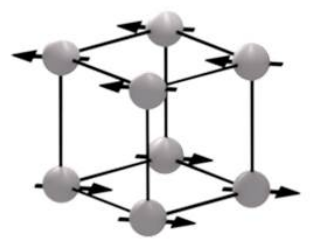

A-AFM

Figure 8. Orbital reconstruction and induced antiferromagnetic (AFM) ordering, due to tetragonal distortion.

When the strain is relatively small and other effects start to play a role, discrepancy arises. This occurs for the LSMO films grown on $\mathrm{NdGaO}_{3}$ (NGO) and STO substrates, where the lattice mismatch is $-0.5 \%$ and $0.6 \%$, respectively. Tebano et al. [34] and Lepetit et al. [37] found that the LSMO film on (001) $\mathrm{SrTiO}_{3}$ substrate will end up with $3 z^{2}-\mathrm{r}^{2}$ orbital ordering at the interface. This counters to the expectation from the tensile strain imposed on LSMO, which flattens the $\mathrm{MnO}_{6}$ octahedra and should favor $x^{2}-y^{2}$ orbital occupation. The unexpected $3 z^{2}-r^{2}$ orbital reconstruction has been proposed to be caused by interface or surface effects, which become prominent in ultrathin LSMO films. First-principle theoretical calculations indicates that the $\mathrm{MnO}_{2}$ - and ( $\left.\mathrm{La}, \mathrm{Sr}\right) \mathrm{O}$-terminated surfaces show strong tendencies to have $3 z^{2}-r^{2}$ and $x^{2}-y^{2}$ orbital reconstructions, respectively [61]. The missing top apical oxygen for the $\mathrm{MnO}_{2}$ termination surface will provide a repulsive potential, and an elongated Mn-O bond along the surface normal direction, downshifting the $3 z^{2}-r^{2}$ orbital. Regarding the (La,Sr)O termination, the $3 z^{2}-r^{2}$ orbital will upward-shift due to strengthened pd $\sigma$ hybridization when the above $\mathrm{MnO}_{2}$ layer is missing. This predicted effect of surface termination on orbital polarization has been confirmed experimentally by Pesquera et al. [52]. In the case of LSMO interfacing empty $3 z^{2}-r^{2}$ material, the Mn $3 z^{2}-r^{2}$ orbital has a large overlap with the empty $3 z^{2}-r^{2}$ through bridging the oxygen at the interface, and it favors delocalization into the empty $3 z^{2}-r^{2}$ orbital. Such a mechanism will lower the $\mathrm{Mn} 3 \mathrm{z}^{2}-\mathrm{r}^{2}$ orbital, and therefore enhance $3 z^{2}-\mathrm{r}^{2}$ orbital occupation at the interface. This has been proposed to explain $3 z^{2}-r^{2}$ orbital reconstruction in ultrathin LSMO on STO, and contributes to the origin of the dead layer $[37,62]$. Though several reports show $3 z^{2}-r^{2}$ orbital reconstruction, linear-dichroism (LD) measurements by Huijben et al. revealed different results that the $\mathrm{x}^{2}-\mathrm{y}^{2}$ orbital is preferentially occupied without any orbital reconstruction at the interface [18]. Their results show very similar LD signals between thick films (e.g., 70 u.c.) and ultrathin films (e.g., 5 u.c.). This fact indicates that the orbital plays a minor role in inducing the dead layer. The proposed interface that enhanced $3 z^{2}-r^{2}$ orbital reconstruction would also suggest a larger $3 z^{2}-r^{2}$ orbital population and reduced magnetism for STO capped LSMO, which do not agree with the experimentally observed reduced dead layer thickness by STO capping [39]. Since the observed orbital polarization in these LSMO films is very small, another open question then arises for whether this small orbital polarization 
is significant enough to unbalance the competition between double exchange and superexchange, and practically cause an AFM ground state.

A systematical investigation of the correlation of orbital polarization with magnetic ordering has been performed on LSMO films grown on NGO (110) substrates [40], as shown in Figure 9. To quantify the orbital polarization, the $M n x^{2}-y^{2}$ and $3 z^{2}-r^{2}$ orbitals were preferentially probed by in-plane $(\sigma)$

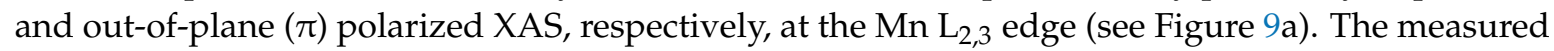
XAS is shown in the top panel of Figure $9 \mathrm{~b}$. For different films, their two spectra $\mathrm{I}(\sigma)$ and $\mathrm{I}(\pi)$ all show slight differences, indicating a small linear dichroism. The X-ray linear dichroism (XLD) is calculated as the intensity difference between the signals with different polarization $(\mathrm{I}(\sigma)-\mathrm{I}(\pi))$. As shown in the bottom panel of Figure 9b, all films exhibited quite similar XLD characteristics, e.g., 6 u.c. LSMO (noted as $\mathrm{L}_{6}$ )- and 9 u.c. STO-buffered 6 u.c. LSMO (noted as $\mathrm{L}_{6} / \mathrm{S}_{9}$ ) on the one hand; $\mathrm{L}_{30}$ and $\mathrm{L}_{30} / \mathrm{S}_{6}$ on the other hand. By integrating the spectra in the range 649-659 eV (around the $\mathrm{Mn} \mathrm{L}_{2}$ edge), the area under the XLD peak (ALD) is obtained and plotted as a function of the Curie temperature $\left(T_{C}\right)$ for different films (see Figure 9c). A positive (or negative) ALD indicates a preferential occupation of the $3 z^{2}-r^{2}\left(\right.$ or $\left.x^{2}-y^{2}\right)$ orbital. The ALDs of all different films are within $\sim 1 \%$, indicating a very small orbital polarization with slightly preferential occupation of the $3 z^{2}-r^{2}$ orbital. Since the XLDs were taken at $300 \mathrm{~K}$, which were well above the $T_{\mathrm{C}}$ of LSMO films that were thinner than 30 u.c. (Figure $9 \mathrm{c}$ ), therefore, the contribution of magnetization to XLD can be ruled out. A change of orbital polarization is not accompanied by any change of the Curie temperature. Therefore, one can infer that the small degree of orbital polarization present plays a minor role in magnetic ordering when the lattice mismatch is relatively small.

(a)

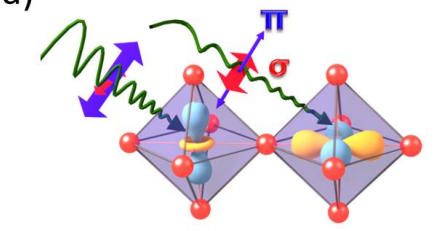

(c)

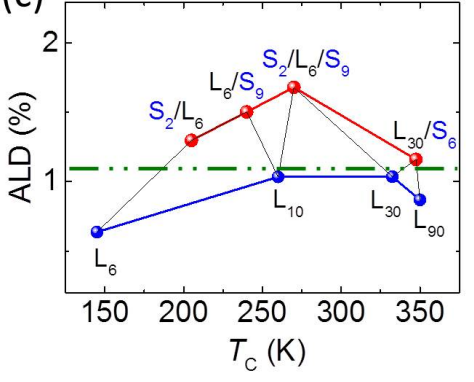

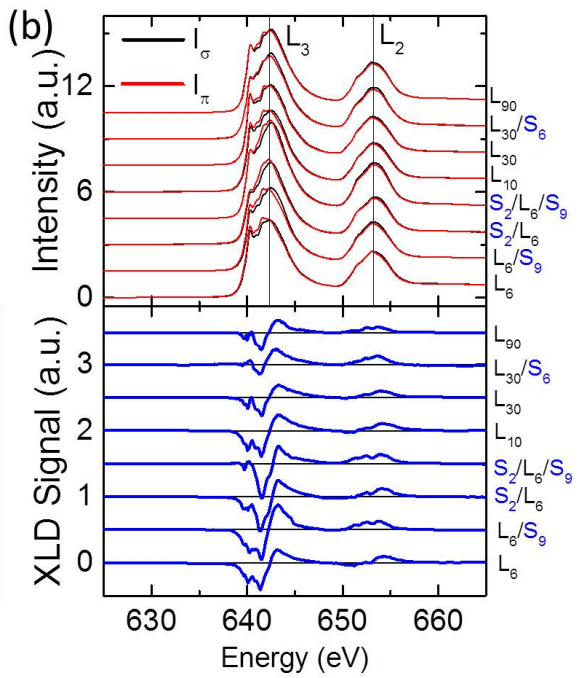

Figure 9. (a), Sketch of the X-ray absorption measurement of $3 z^{2}-r^{2}$ and $x^{2}-y^{2}$ orbital occupations by $\pi$ and $\sigma$-polarized X-rays, respectively. (b), Polarized X-ray absorption (XAS) of the $\mathrm{L}_{2,3}$ edge (top panel) and the corresponding linear dichroism (bottom panel) of different LSMO films with/without the STO buffer/capping layer. (c), Integrated linear dichroism from $649-659 \mathrm{eV}$ normalized by the $\left(\mathrm{I}_{\sigma}+\mathrm{I}_{\pi}\right)$ for LSMO films, ALD $=\left(I_{\sigma}-I_{\pi}\right) /\left(I_{\sigma}+I_{\pi}\right) \times 100 \%$. All X-ray linear dichroism (XLDs) were measured at $300 \mathrm{~K}$. Here, $\mathrm{S}$ and L in the sample labels denote STO and LSMO, respectively. Reproduced with permission from Liao et al. [40]. Copyright 2017 John Wiley and Sons.

It is noteworthy that the 6 u.c. LSMO had a smaller orbital polarization than thicker films. This indicates a competitive action of compressive strain and octahedral tilt on the tetragonal distortions of the octahedra. Since the Mn-Mn distances in the (110) plane are locked-in by the NGO substrate, the tilting of the octahedra will increase the $\mathrm{Mn}-\mathrm{O}$ bond length in the (110) plane, compensating the effect from compressive strain which tends to elongate the $\mathrm{Mn}-\mathrm{O}$ bond in the direction normal to the interface. This effect subsides with increasing thickness, and hence orbital polarization will increase in 
thicker films. With a STO buffer layer, the octahedral tilt disappears in ultrathin LSMO [63], squeezing the in-plane $\mathrm{Mn}-\mathrm{O}$ bond, resulting in an increased occupation of the $3 z^{2}-r^{2}$ orbital in $\mathrm{L}_{6} / \mathrm{S}_{9}$ film as shown in Figure 9c. The STO capping layer is also found to enhance orbital polarization of both the non-buffered and buffered 6 u.c. LSMO, indicating a bulk-like electronic structure at the surface layer, which can be connected to the recovery of surface magnetism, as illustrated by RXR (see Figure 6).

\subsection{Valence Change}

According to the phase diagram of bulk $\mathrm{La}_{1-x} \mathrm{Sr}_{x} \mathrm{MnO}_{3}$ (see Figure 1b), the ground state strongly depends on the Sr doping level. The $\mathrm{La}_{1-x} \mathrm{Sr}_{x} \mathrm{MnO}_{3}$ is metallic when the doping is in the range $0.2<x<0.5$. There is a narrow range $0.15<x<0.2$ where the $\mathrm{La}_{1-x} \mathrm{Sr}_{x} \mathrm{MnO}_{3}$ is a ferromagnetic insulator, making the doping range $(0.15<x<0.5)$ for the ferromagnetic ground state a little larger than that for the metallic phase. The very explicit consequence of the hole doping by $\mathrm{Sr}$ is the increasing valence from $\mathrm{Mn}^{3+}$ to $\mathrm{Mn}^{3+x}$. This fact may suggest that another mechanism is responsible for the origin of the dead layer: a change of Mn valence in LSMO. A change of Mn valence can be caused by different reasons: (I) polar catastrophe-induced charge transfer or oxygen vacancy formation [38,55,56]; (II) Sr-segregation [46-49]; (III) interface diffusion [44,49,50].

The polar catastrophe model proposed by Boschker et al. [56] is shown in Figure 10. The LSMO is a polar material with an alternate stacking of charge planes of $(\mathrm{La}, \mathrm{Sr}) \mathrm{O}^{0.67}$ and $\mathrm{MnO}_{2}{ }^{-0.67}$. No matter grown on $\mathrm{SrO}$-termination $\mathrm{STO}$ or $\mathrm{TiO}_{2}$ termination $\mathrm{STO}$, a potential is built in from interface to surface due to polar discontinuity across LSMO/STO interface (see Figure 10a,b). This built-in potential results in charge instability and certain amount of electrons have to be transferred from the surface to the interface, to remove the built-in potential. Such mechanism will electron-dope the interface, and therefore deteriorate the properties. If this is the case, inserting another polar material $\left(\mathrm{La}_{0.33} \mathrm{Sr}_{0.67} \mathrm{TiO}_{3}\right)$ at the interface (Figure 10c,d); cancelling this effect should have significant consequences on the transport properties. Indeed, Boschker et al. have demonstrated enhanced conductivity and magnetization by such interface charge engineering (see Figure 10e,f). For example, non-interface-engineered (nIE) 6 u.c. LSMO is insulating, but interface-engineered (IE) 6 u.c. LSMO is metallic.

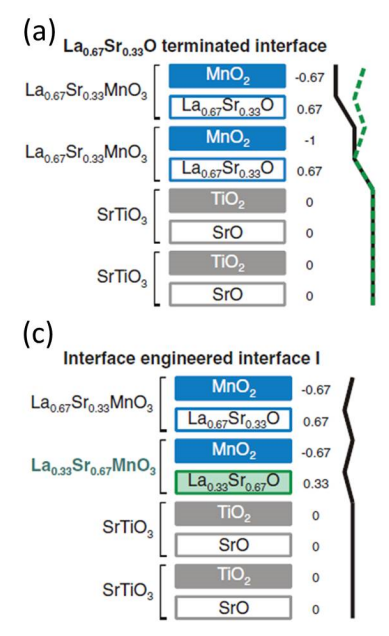

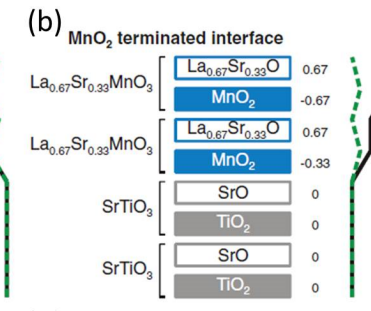

(d)

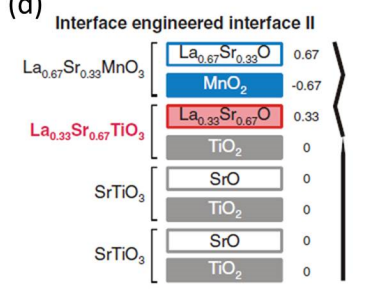

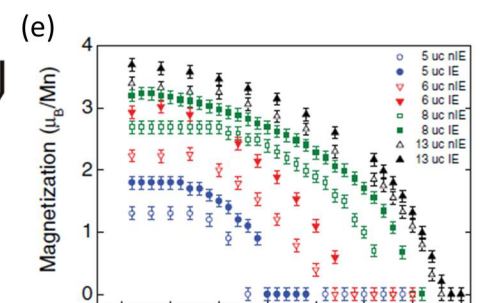

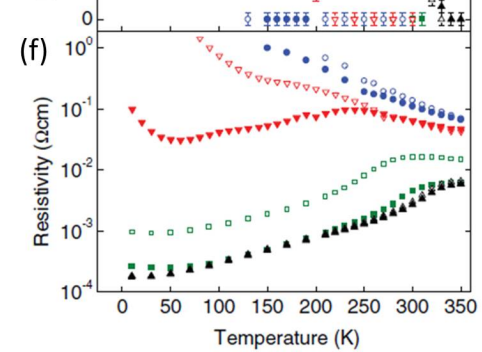

Figure 10. Polar catastrophe model of LSMO film on STO. The termination of the STO substrate is $\mathrm{TiO}_{2}$ in $(\mathbf{a}, \mathbf{c})$, and is $\mathrm{SrO}$ in $(\mathbf{b}, \mathbf{d})$. The black line and green dotted line indicate the electrostatic potential profile before and after charge transfer, respectively. Comparison of (e) magnetization and (f) transport properties between non-interface-engineered (nIE) and interface-engineered (IE) LSMO films. Reproduced with permission from Boschker et al. [56]. Copyright 2012 John Wiley and Sons.

Microscopic investigation of the polar catastrophe model has been done by using atomic resolved electron energy loss spectra (EELS) performed in a STEM [55]. The layer-resolved valence state was 
obtained for different doping $\mathrm{La}_{1-x} \mathrm{Sr}_{x} \mathrm{MnO}_{3}(x=0-0.5)$ grown on STO. From the valence profile, Mundy et al. concluded that the enrichment of electrons in LSMO near the interface, and the extra electrons at the interface, quantitatively agree with the predicted charge $\left(\mathrm{q}=(x / 2-1 / 2) \mathrm{e}^{-}\right)$that is needed to cancel the polar catastrophe for insulating $\mathrm{La}_{1-x} \mathrm{Sr}_{x} \mathrm{MnO}_{3}$. However, the transfer charge dramatically drops when $\mathrm{La}_{1-x} \mathrm{Sr}_{x} \mathrm{MnO}_{3}$ is metallic, due to the onset of screening effects. For $x=0.3$, the total transfer charge is around $0.1 \mathrm{e}^{-}$. This fact suggests the overestimation of the charge transfer due to polar discontinuity, and its effect on LSMO magnetization and transport properties, since the extra $0.1 \mathrm{e}^{-}$that which varies the Mn valence to $3.23^{+}$at upper limit is not large enough to move the LSMO into antiferromagnetic ground state (see Figure 1b). The spreading of the charge in space would reduce the change of the Mn valence. Different from EELS, in situ photoemission and low energy electron diffraction measurements suggest another scenario, where spreading oxygen vacancies are formed near the top surface, and electrons are distributed near the bottom of the interface, which induces an opposite field to compensate the polar field [38].

Another source of valence change is $\mathrm{Sr}$ segregation. The electrostatic interaction will make the polar surface unstable. However, it can be stabilized by neutralizing the surface, through Sr-segregation. Sr-segregation will lead to a neutral SrO layer at the surface, and the formation of $\mathrm{Mn}^{4+}$, degrading the LSMO into the G-type antiferromagnetic insulator region. The Sr-segregation has been confirmed by angle resolved XPS and surface X-ray diffraction [47-49], and is now revisited by STEM [49].

\subsection{Orbital Hybridization}

While the orbital reconstruction and valence change are the two focused models explaining the origin of the dead layer, another mechanism of reduced bandwidth-driven metal-to-insulator transition in ultrathin LSMO has been proposed recently [40]. The role of bandwidth in determining the electronic ground state in LSMO has been demonstrated long time ago in bulk LSMO [64]. The relation between the bandwidth and the bond angle can be described by an empirical formula, $w \propto \frac{\cos (\pi-\theta) / 2}{d^{3.5}}$ [65]. Here, $d$ is the Mn-O bond length, and $\theta$ is the Mn-O-Mn bond angle. By isovalent substitution of the A site with different rare earth elements for obtaining different structure distortions while maintaining the same charge doping level, Garcia-Munoz et al. demonstrated that the $T_{C}$ decreases with a reducing Mn-O-Mn bond angle [64].

For the LSMO thin film, more and more experiments indicate that the octahedra has different rotation patterns near the interface and away from the interface, due to interfacial oxygen octahedral coupling (OOC) $[40,63,66,67]$. The interfacial OOC rearranges the octahedral tilting, to retain oxygen octahedral connectivity at the interface between two dissimilar perovskite oxides. X-ray diffraction of LSMO on STO revealed a gradual evolution of the octahedral tilt pattern from the interface, to a distant interface region [66]. At the LSMO/STO interface, the tilting disappears, and LSMO becomes $\mathrm{a}^{0} \mathrm{a}^{0} \mathrm{a}^{0}$ in Glazer notation [68]. Away from STO, the tilt pattern becomes $\mathrm{a}^{+} \mathrm{a}^{-} \mathrm{c}^{0}$, and the tilt angle gradually relaxes. Thanks to the advanced annular bright field (ABF) technique, oxygen atoms can be spatially resolved in STEM images, allowing us to visualize the octahedral tilt at the atomic scale. Using the $\mathrm{ABF}$ technique, the change of the octahedral tilt near the interface has been confirmed microscopically $[40,63,67]$. One example of the visualization of the octahedra tilt across the LSMO/NGO interface is shown in Figure 11. A clearly enhanced octahedral tilt of LSMO is observed at the LSMO/NGO interface. The profile of the tilt angle $\beta$, as shown in Figure 11b, further illustrates the large degree of tilting near the interface, and the tilting angle gradually increases when further away from the interface region. With inserting an STO buffer layer to locally engineer the OOC, the tilt in the LSMO near the interface then can be dramatically reduced (see Figure 11a,b). A direct link between the bond angle and the ferromagnetic ordering is shown in Figure 11c. For non-STO-buffered LSMO, $T_{\mathrm{C}}$ is $150 \mathrm{~K}$, which is promoted to $180 \mathrm{~K}$ and $250 \mathrm{~K}$ by 1 u.c. and 9 u.c. STO buffer layers, respectively. This strongly suggests the impact of octahedral tilting, and therefore the orbital hybridization on the ferromagnetic ordering. 
(a)

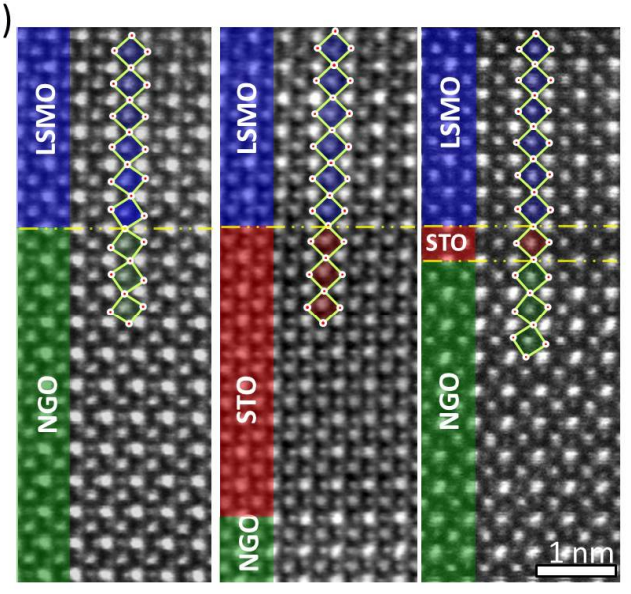

(b)

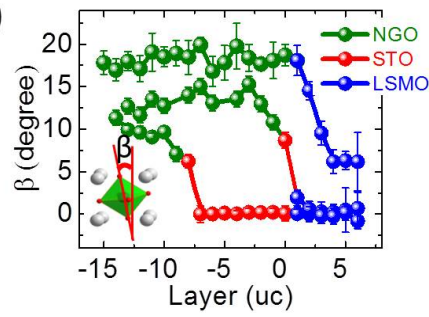

(c)

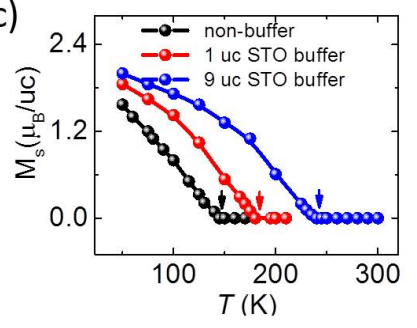

Figure 11. (a) Inversed annular bright-field STEM images of (left panel) $\mathrm{LSMO} / \mathrm{NdGaO}_{3}(\mathrm{LSMO} / \mathrm{NGO})$, (middle panel) LSMO/STO (9 u.c.)/NGO and (right panel) LSMO/STO (1 u.c.)/NGO heterostructures. The connectivity of oxygen octahedra across the interfaces is indicated. (b) Layer-position dependent mean octahedral tilt angle $(\beta)$, together with their standard deviation in LSMO/NGO heterostructures with and without a STO buffer layer. The data for non-buffered sample is shifted upward by $6^{\circ}$ for clarity. (c) The temperature-dependent saturated magnetization for buffered and non-STO-buffered LSMO films. All the LSMO films are 6 u.c. thick. Reproduced with permission from Liao et al [40]. Copyright 2017 John Wiley and Sons. Reproduced with permission from Liao et al. [63]. Copyright 2016 Nature Publishing.

Experimentally, the effect of the octahedral tilt on the strength of p-d hybridization is directly measured by atomically resolved fine-structure changes at the O-K edge in EELS. Figure 12 shows layer resolved spectra of the O-K edge spectrum taken from the individual layers across a LSMO film grown on NGO substrate. The pre-edge peak located near $530 \mathrm{eV}$ arises from the mixture of O-2p and Mn-3d character $[69,70]$. Getting closer to the LSMO/NGO interface, the pre-peak intensity is found to be smaller, suggesting weaker O-2p and Mn-3d hybridization at the interface. This smaller $\mathrm{Mn}-\mathrm{O}$ orbital hybridization is consistent with the smaller Mn-O-Mn bond angle near the interface. The change of the orbital hybridization from the bulk region towards the interface agrees well with the schematic structure profile shown in Figure 12a.
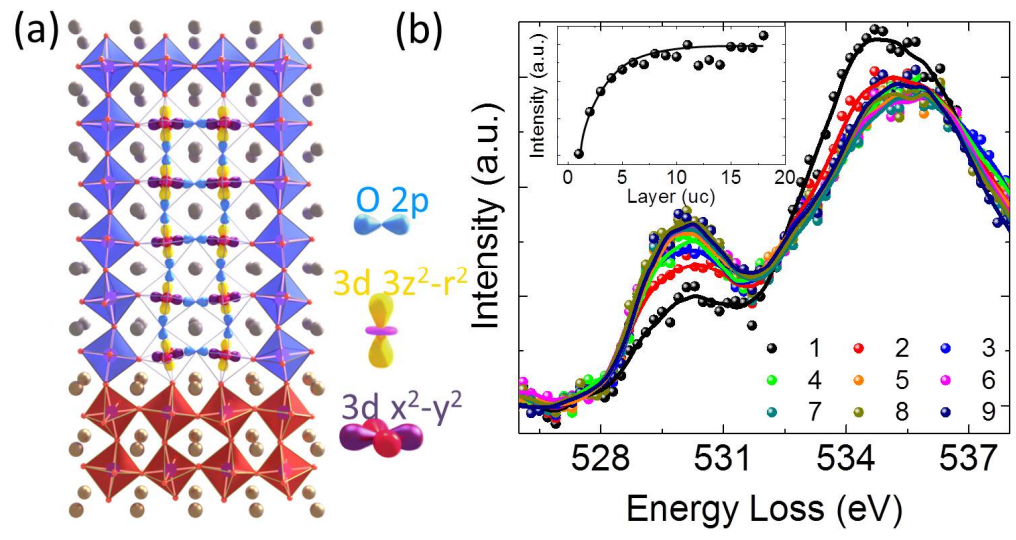

Figure 12. (a) Schematic view of the evolution of the overlap of Mn-3d orbital with O-2p orbitals across the interface in the oxide heterostructure. (b) Detailed O-K edge spectra of individual layers from atomically-resolved electron energy loss spectra (EELS) mapping for a 30 u.c. LSMO film on NGO substrate. Reproduced with permission from Liao et al. [40]. Copyright 2017 John Wiley and Sons. 


\subsection{Anderson Localization Effect}

The electric dead layer is normally thicker than the magnetization dead layer. In other words, we have a ferromagnetic insulator (FMI) phase in ultrathin LSMO. This is not consistent with the double exchange mechanism which couples the ferromagnetism to metallicity, as in bulk LSMO. Transport analysis shows that the anomalous FMI phase is due to the Anderson localization effect $[39,57,71]$. As shown in Figure 13, the temperature-dependent resistivity indicates that LSMO films on STO gradually evolve from weak localization to strong localization, with reducing thickness. For metallic film $\left(t>t_{c}\right)$, the resistivity linearly depends on $\log [T]$, as shown in Figure 13a,b. This is a strong signature of the weak localization where enhanced backscattering needs to be considered with the presence of impurities [72]. While at critical thickness, the transport does not follow the weak localization behavior (see Figure 13c); instead, the ultrathin critical film follows the Mott's variable range hopping (VRH), $\rho=\rho_{0} \exp \left(T_{0} / T\right)^{\alpha}$ with exponent $\alpha=1 / 3$, as expected in two dimensions (see Figure 13d) [73]. For thinner films $\left(t<t_{c}\right)$, the films enter into the thermal activation region and the resistivity can be described by an Arrhenius function $\left(\rho=\rho_{0} e^{-\Delta E / k T}\right)$ (see inset in Figure 13c). The transition from weak to strong localization is characterized by the Ioffe-Regel (IR) limit of resistivity [72] above which the system is turned into a strong localization region. For a 2D system, the IR limit is related to the sheet resistance of $\mathrm{R}_{\mathrm{IR}} \approx 25 \mathrm{~K} \Omega$. As shown in Figure 13e, we can find metallic films with weak localization when the resistivity of the LSMO films on STO (001) is below the IR limit, while above the IR limit, the films are nonmetallic. The characteristic transport properties indicate that the thickness driven metal-nonmetal transition is associated with a 3D metallic state to a 2D insulating state-dimensional cross-over transition. The carrier localization likely arises from an intrinsic A-site cation disorder, or/and residual oxygen vacancies $[39,57,74]$. Suppressed localization effect and reduced critical thickness have been realized by minimizing the oxygen vacancies concentration [39,57]. Since a perfect crystal is not realistic, this disorder-induced localization serves as the universal driving force for the MIT in many ultrathin film oxide materials.
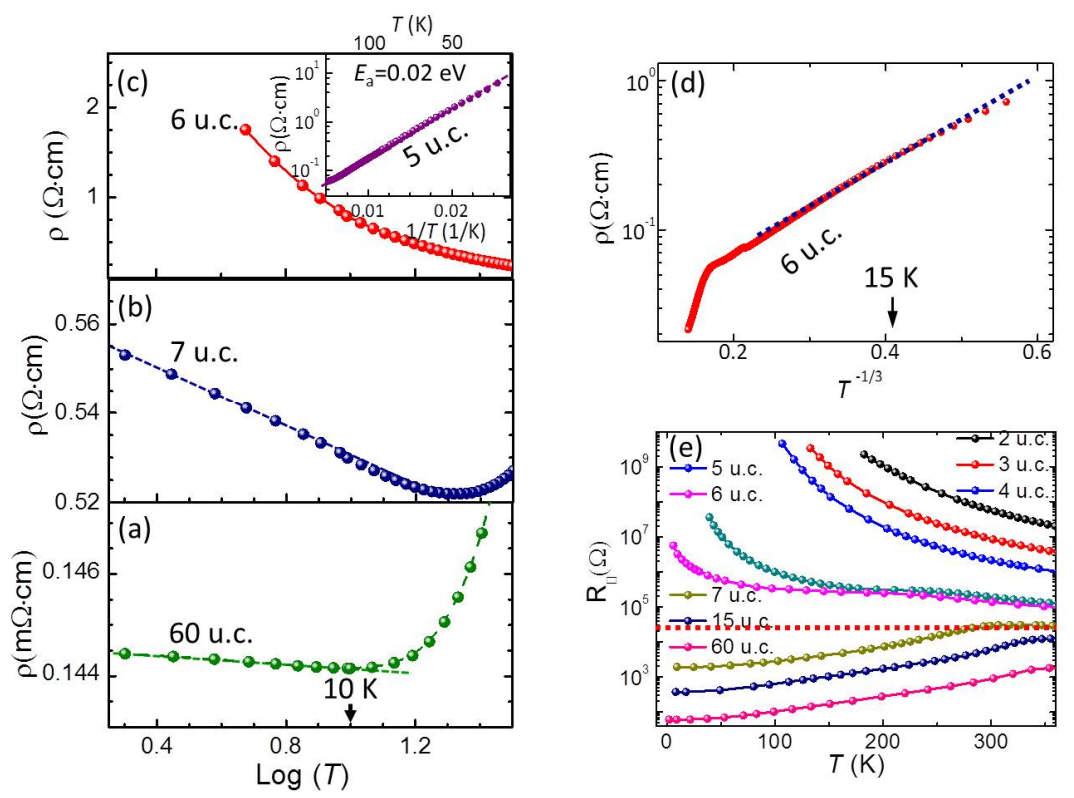

Figure 13. The resistivity versus $\log (T)$ for (a) 60 , (b) 7 , and (c) 6 u.c. LSMO films on STO (001). The inset in (c) shows the logarithmic plot of resistivity of a function of $1 / T$ for 5 u.c. film. (d) Logarithmic plot of resistivity as a function of $1 / T^{1 / 3}$ for 6 u.c. LSMO. (e) $T$ dependence of the sheet resistance for different thicknesses of LSMO films. The red dotted line indicates the Ioffe-Regel (IR) limit for the onset of strong localization. Figure is adapted from Ref. [39]. 


\subsection{Structure Imperfection}

As mentioned above, extrinsic ingredients, such as oxygen vacancies or loss of cations, which arises from the non-optimal growth process, can vary the dead layer thickness as well. Regarding PLD growth, the oxygen partial pressure, laser fluence, and growth temperature can play a role in affecting the film quality. It is well known that insufficient oxidized environment, e.g., low oxygen partial pressure will lead to oxygen vacancies formation. Besides, the laser fluence is also found to influence the film quality. By varying the laser spot size while maintaining the laser power to grow different LSMO/STO superlattices, Kourkoutis et al. demonstrate that extended defects are formed in LSMO layers and the interfaces between LSMO/STO become more diffuse as the spot size becomes smaller [50], as shown in Figure 14. More detailed EELS analysis shows that some of the B-sites are locally filled by $\mathrm{Sr}$ atoms, causing the A-site/B-site cation ratio in LSMO to be excess of one in the cases that samples were grown at high laser fluence (see Figure 14e-g). These excess $\mathrm{Sr}$ atoms form rock-salt-type layers (see Figure 14h). The off-stoichiometry and local impurity phase deteriorate the magnetization and transport, leading to suppressed magnetization and insulating behavior in ultrathin LSMO films.

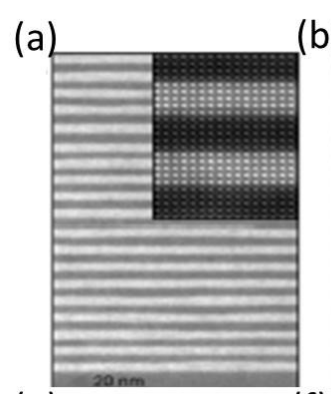

(e)

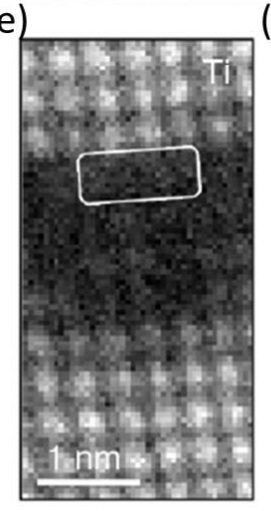

(b)

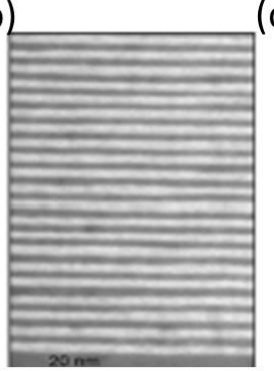

(f)

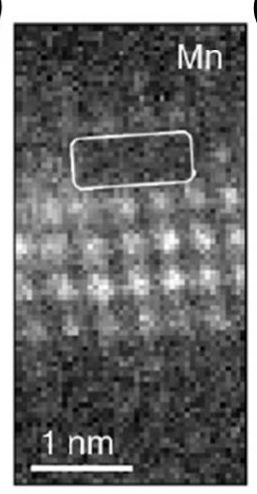

(c)

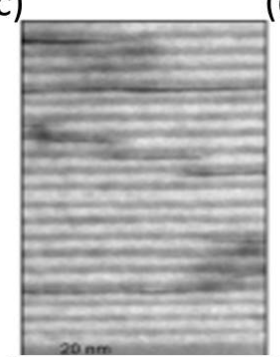

(g)

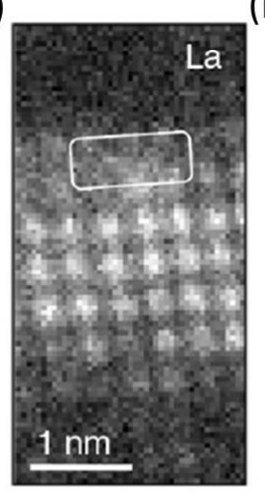

(d)

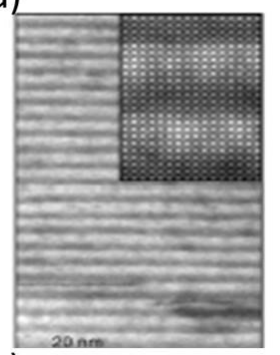

(h)

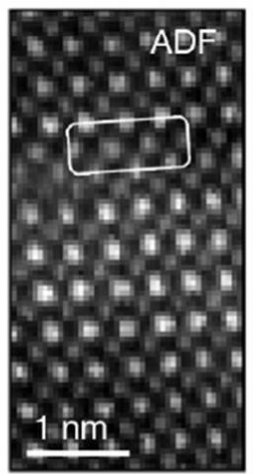

Figure 14. Annular dark field image of the cross-section of $\left(\mathrm{La}_{0.7} \mathrm{Sr}_{0.3} \mathrm{MnO}_{3}\right)_{5} /\left(\mathrm{SrTiO}_{3}\right)_{5}$ superlattices grown at different laser spot size (a) 10.2, (b) 7.5 , (c) 5 and (d) $1.6 \mathrm{~mm}^{2}$. The laser energy during growth was fixed at $35 \mathrm{~mJ}$. (e-g) are the elemental mapping of (e) Ti, (f) Mn, and (g) La for LSMO grown at a laser spot size of $1.6 \mathrm{~mm}^{2}$. (h) Simultaneously recorded annular dark field image. Figures are adapted from Ref. [50].

\subsection{Overview of the Mechanism of MIT in LSMO}

Many models have been proposed to explain the origin of thickness-driven MIT in LSMO, Though the debates still remain, the proposed models and related experiment evidences give us quite different aspects to look into the underlying physics, and imply the cooperative effects on the dead layer. The extrinsic defects (oxygen vacancies, dislocations, etc.) induced by growth generally destroy the long range magnetic order, and thus increases the dead layer thickness. The strain, which is heavily investigated in the past, is another important effect that will degrade the properties especially for ultrathin films. However, in the situation of small strain, which can only result in minor orbital reconstruction, the octahedral rotation pattern near the interface modified by interface octahedral 
coupling emerges to be a key ingredient that determines both the magnetic and transport properties. In terms of a widely observed dead layer in many metallic oxides, as discussed in next section, the dimensionality effect is also believed to play a vital role, though the pure dimensionality effect is hard to obtain in real films, due to the unavoidable interface effect, stain, and broken symmetry. More experimental and theoretical efforts are still required to eventually figure out the origin of the dead layer. For examples, some dynamic strategies to control the MIT, such as charge modulation by the external electric field [75,76], may help us to separately understand the individual effects on MIT, and pave a new path to understand the physics of MIT in LSMO. Developing a method to fabricate a freestanding ultrathin LSMO is also highly desired in the future, in order to really gain an insight into the dimensionality effect [77].

\section{MIT in Other Correlated $\mathrm{ABO}_{3}$ Thin Films}

Besides LSMO, reduced dimensionality-driven MIT has been broadly observed in many other correlated metal oxides, such as $\mathrm{LNO}[14,78,79], \mathrm{SrVO}_{3}$ (SVO) [15] and $\mathrm{SrRuO}_{3}$ (SRO) [16], and $\mathrm{SrIrO}_{3}$ (SIO) $[80,81]$. The dead layer thicknesses of these oxide materials are also quite similar. All the LNO, $\mathrm{SVO}, \mathrm{SRO}, \mathrm{LSMO}$, and SIO share the same dead layer thickness of $\sim 3$ u.c. This similarity may also suggest that the dead layer has a common origin. Similar to the capping effect on LSMO $[39,40]$, the surface capping has been found to decrease the dead layer thickness of both LNO [78] and SRO [82]. The capping layer plays a role in reducing the surface structure distortion [78]. The MIT in LNO ultrathin films also exhibit dimensional-crossover from weak localization to strong localization [79]. With reducing dimensionality, the electron has shorter scattering path and the Anderson localization effect becomes stronger, therefore the Anderson localization effect is amplified in ultrathin films. This unavoidable effect initiated by inherent disorder will prevent the thin films from exhibiting metallic behavior. Another possible common reason that is responsible for the dead layer may be the enlarged electron-electron correlation effect with reduced dimensionality. An Increased electron correlation tends to localize the electron, and induce a Mott insulator state. Though the true mechanism is still a question, due to the intrinsic complexity in these correlated materials, due to the delicate coupling between multiple degrees of freedom of spin, charge, orbital, and lattice, the common dead layer behavior in different correlated oxides strongly implies an important role of the reduced dimensionality effect.

\section{Future Prospect}

In summary, a comprehensive study of the dead layer of LSMO reveals the important role of intimate coupling between lattice, charge, orbital, and spin, in the transport and magnetism of LSMO. These couplings are sensitive to off-stoichiometry, orbital reconstruction, change of bandwidth, and Mn valence, leading to suppressed magnetism and insulating ground state. Besides, the disorder-induced electronic localization, which is amplified by reduced dimensionality, further prevents the ultrathin film from exhibiting metallic states, setting up an ultrathin limit of the dead layer. However, some questions or debates still remain. What is the actual physical consequence of the polar catastrophe due to the polar nature of LSMO? Is it the formation of oxygen vacancies or pure charge transfer? Can the observed small orbital polarization really can destroy the ferromagnetic ordering, and is it therefore is vital to induce the dead layer?

The key to understand the properties of the ultrathin films will be advanced techniques that can probe materials at the atomic scale. Recently developed techniques such as STEM, PNR, and $\mathrm{RXR}$, which can provide very local lattice, spin, orbital configuration, and electronic structure, may eventually reveal the origin of the thickness-driven metal-to-insulator transition and the dead layer. In this regard, we are optimistic to see more breakthroughs in the near-future to understand the dead layer by atomically resolving all of the orbital, spin, lattice and charge structures in the films.

The understanding and control of the dead layer are also very attractive for technological applications, such as transistors, memory devices, and sensors. At the verge of the metal-to-insulator 
transition, external stimulation, e.g., the magnetic field, field gating, elastic strain, or pressure, could switch the ultrathin layers from metal to insulator, or the other way around. A thin film with critical thickness can be very susceptible to external stimulations, and it therefore serves as a good material system for fabricating electronic devices. In this regard, more research on the control of the dead layers should be required.

Author Contributions: Writing-Original Draft Preparation: Z.L. and J.Z.; Writing-Review \& Editing: Z.L. and J.Z.

Funding: Research by Z.L. was funded by Hundred Talent Program of the Chinese Academy of Science. Research by J.Z. was funded by U.S. DOE under Grant No. DOE DE-SC0002136.

Conflicts of Interest: The authors declare no conflict of interest.

\section{References}

1. Smith, H.M.; Turner, A.F. Vacuum deposited thin films using a ruby laser. Appl. Opt. 1965, 4, 147-148. [CrossRef]

2. Christen, H.M.; Eres, G. Recent advances in pulsed-laser deposition of complex oxides. J. Phys. Condens. Matter 2008, 20, 264005. [CrossRef] [PubMed]

3. Schlom, D.G. Perspective: Oxide molecular-beam epitaxy rocks! APL Mater. 2015, 3, 62403. [CrossRef]

4. Schlom, D.G.; Chen, L.-Q.; Eom, C.-B.; Rabe, K.M.; Streiffer, S.K.; Triscone, J.-M. Strain tuning of ferroelectric thin films. Ann. Rev. Mater. Res. 2007, 37, 589-626. [CrossRef]

5. Cao, J.B.; Wu, J.Q. Strain effects in low-dimensional transition metal oxides. Mater. Sci. Eng. R 2011, 71, 35-52. [CrossRef]

6. Hwang, H.Y.; Iwasa, Y.; Kawasaki, M.; Keimer, B.; Nagaosa, N.; Tokura, Y. Emergent phenomena at oxide interfaces. Nat. Mater. 2012, 11, 103. [CrossRef]

7. Zubko, P.; Gariglio, S.; Gabay, M.; Ghosez, P.; Triscone, J.-M. Interface Physics in Complex Oxide Heterostructures. Annu. Rev. Condens. Matter Phys. 2011, 2, 141. [CrossRef]

8. Lee, H.N.; Christen, H.M.; Chisholm, M.F.; Rouleau, C.M.; Lowndes, D.H. Strong polarization enhancement in asymmetric three-component ferroelectric superlattices. Nature 2005, 433, 395. [CrossRef]

9. Disa, A.S.; Kumah, D.P.; Malashevich, A.; Chen, H.H.; Arena, D.A.; Specht, E.D.; Ismail-Beigi, S.; Walker, F.J.; Ahn, C.H. Orbital engineering in symmetry-breaking polar heterostructures. Phys. Rev. Lett. 2015, 114, 026801. [CrossRef]

10. Boris, A.V.; Matiks, Y.; Benckiser, E.; Frano, A.; Popovich, P.; Hinkov, V.; Wochner, P.; Castro-Colin, M.; Detemple, E.; Malik, V.K.; et al. Dimensionality control of electronic phase transitions in nickel-oxide superlattices. Science 2011, 332, 937-940. [CrossRef]

11. Guo, H.W.; Wang, Z.; Dong, S.; Ghosh, S.; Saghayezhian, M.; Chen, L.; Weng, Y.; Herklotz, A.; Ward, T.Z.; Jin, R.Y.; et al. Interface-induced multiferroism by design in complex oxide superlattices. Proc. Natl. Acad. Sci. USA 2017, 114, 5062. [CrossRef] [PubMed]

12. Kim, T.H.; Puggioni, D.; Yuan, Y.; Xie, L.; Zhou, H.; Campbell, N.; Ryan, P.J.; Choi, Y.; Kim, J.-W.; Patzner, J.R.; et al. Polar metals by geometric design. Nature 2016, 533, 68. [CrossRef] [PubMed]

13. Yadav, A.K.; Nelson, C.T.; Hsu, S.L.; Hong, Z.; Clarkson, J.D.; Schlepütz, C.M.; Damodaran, A.R.; Shafer, P.; Arenholz, E.; Dedon, L.R.; et al. Observation of polar vortices in oxide superlattices. Nature 2016, 11, 198. [CrossRef] [PubMed]

14. King, P.D.C.; Wei, H.I.; Nie, Y.F.; Uchida, M.; Adamo, C.; Zhu, S.; He, X.; Božović, I.; Schlom, D.G.; Shen, K.M. Atomic-scale control of competing electronic phases in ultrathin $\mathrm{LaNiO}_{3}$. Nat. Nanotechnol. 2014, 9, 443. [CrossRef] [PubMed]

15. Yoshimatsu, K.; Okabe, T.; Kumigashira, H.; Okamoto, S.; Aizaki, S.; Fujimori, A.; Oshima, M. Dimensional-crossover-driven metal-insulator transition in $\mathrm{SrVO}_{3}$ ultrathin films. Phys. Rev. Lett. 2010, 104, 147601. [CrossRef] [PubMed]

16. Xia, J.; Siemons, W.; Koster, G.; Beasley, M.R.; Kapitulnik, A. Critical thickness for itinerant ferromagnetism in ultrathin films of $\mathrm{SrRuO}_{3}$. Phys. Rev. B 2009, 79, 140407R. [CrossRef]

17. Koster, G.; Klein, L.; Siemons, W.; Rijnders, G.; Dodge, J.S.; Eom, C.-B.; Blank, D.H.A.; Beasley, M.R. Structure, physical properties, and applications of $\mathrm{SrRuO}_{3}$ thin films. Rev. Mod. Phys. 2012, 84, 253. [CrossRef] 
18. Huijben, M.; Martin, L.W.; Chu, Y.-H.; Holcomb, M.B.; Yu, P.; Rijnders, G.; Blank, D.H.A.; Ramesh, R. Critical thickness and orbital ordering in ultrathin $\mathrm{La}_{0.7} \mathrm{Sr}_{0.3} \mathrm{MnO}_{3}$ films. Phys. Rev. B 2008, 78, 094413. [CrossRef]

19. Wang, X.R.; Li, C.J.; Paudel, T.R.; Leusink, D.P.; Hoek, M.; Poccia, N.; Vailionis, A.; Venkatesan, T.; Coey, J.M.D.; Tsymbal, E.Y.; Ariando, H.H. Imaging and control of ferromagnetism in $\mathrm{LaMnO}_{3} / \mathrm{SrTiO}_{3}$ heterostructures. Science 2015, 349, 716-719. [CrossRef]

20. Junquera, J.; Ghosez, P. Critical thickness for ferroelectricity in perovskite ultrathin films. Nature 2003, 422, 506. [CrossRef]

21. Hemberger, J.; Krimmel, A.; Kurz, T.; von Nidda, H.-A.K.; Ivanov, V.Y.; Mukhin, A.A.; Balbashov, A.M.; Loidl, A. Structural, magnetic, and electrical properties of single-crystalline $\mathrm{La}_{1-x} \mathrm{Sr}_{x} \mathrm{MnO}_{3}(0.4<x<0.85)$. Phys. Rev. B 2002, 66, 094410.

22. Tokura, Y.; Tomioka, Y. Colossal magnetoresistive manganites. J. Magn. Magn. Mater. 1999, 200, 1. [CrossRef]

23. Park, J.-H.; Vescovo, E.; Kim, H.-J.; Kwon, C.; Ramesh, R.; Venkatesan, T. Direct evidence for a half-metallic ferromagnet. Nature 1998, 392, 794. [CrossRef]

24. Majumdar, S.; van Dijken, S. Pulsed laser deposition of $\mathrm{La}_{1-x} \mathrm{Sr}_{x} \mathrm{MnO}_{3}$ :thin-film properties and spintronic applications. J. Phys. D Appl. Phys. 2015, 47, 034010. [CrossRef]

25. Xiong, Z.H.; Wu, D.; Vardeny, Z.V.; Shi, J. Giant magnetoresistance in organic spin-valves. Nature 2004, 427, 821. [CrossRef] [PubMed]

26. Chung, B.W.; Brosha, E.L.; Garzon, F.H.; Raistrick, I.D.; Houlton, R.J.; Hawley, M.E. Growth of highly oriented $\mathrm{La}_{0.84} \mathrm{Sr}_{0.16} \mathrm{MnO}_{3}$ perovskite films. J. Mater. Res. 1995, 10, 2518-2522. [CrossRef]

27. Kwon, C.; Robson, M.C.; Kim, K.-C.; Gu, J.Y.; Lofland, S.E.; Bhagat, S.M.; Trajanovic, Z.; Rajeswari, M.; Venkatesan, T.; Kratz, A.R.; et al. Stress-induced effects in epitaxial $\left(\mathrm{La}_{0.7} \mathrm{Sr}_{0.3}\right) \mathrm{MnO}_{3}$ films. J. Magn. Magn. Mater. 1997, 172, 229-236. [CrossRef]

28. Suzukia, Y.; Hwang, H.Y.; Cheong, S.-W.; van Dover, R.B. The role of strain in magnetic anisotropy of manganite thin films. Appl. Phys. Lett. 1997, 71, 140-142. [CrossRef]

29. Bowen, M.; Bibes, M.; Barthélémy, A.; Contour, J.-P.; Anane, A.; Lemaítre, Y.; Fert, A. Nearly total spin polarization in $\mathrm{La}_{2 / 3} \mathrm{Sr}_{1 / 3} \mathrm{MnO}_{3}$ from tunneling experiments. Appl. Phys. Lett. 2003, 82, 233-235. [CrossRef]

30. Lu, Y.; Li, X.W.; Gong, G.Q.; Xiao, G.; Gupta, A.; Lecoeur, P.; Sun, J.Z.; Wang, Y.Y.; Dravid, V.P. Large magnetotunneling effect at low magnetic fields in micrometer-scale epitaxial $\mathrm{La}_{0.67} \mathrm{Sr}_{0.33} \mathrm{MnO}_{3}$ tunnel junctions. Phys. Rev. B 1996, 54, 8357. [CrossRef]

31. Sun, J.Z.; Abraham, D.W.; Rao, R.A.; Eom, C.B. Thickness-dependent magnetotransport in ultrathin manganite films. Appl. Phys. Lett. 1999, 74, 3017-3019. [CrossRef]

32. Borges, R.P.; Guichard, W.; Lunney, J.G.; Coey, J.M.D.; Ott, F. Magnetic and electric "dead" layers in $\left(\mathrm{La}_{0.7} \mathrm{Sr}_{0.3}\right) \mathrm{MnO}_{3}$ thin films. J. Appl. Phys. 2001, 89, 3868-3873. [CrossRef]

33. Angeloni, M.; Balestrino, G.; Boggio, N.G.; Medaglia, P.G.; Orgiani, P.; Tebano, A. Suppression of the metal-insulator transition temperature in thin films. J. Appl. Phys. 2004, 96, 6387-6392. [CrossRef]

34. Tebano, A.; Aruta, C.; Medaglia, P.G.; Tozzi, F.; Balestrino, G.; Sidorenko, A.A.; Allodi, G.; De Renzi, R.; Ghiringhelli, G.; Dallera, C.; et al. Strain-induced phase separation in $\mathrm{La}_{0.7} \mathrm{Sr}_{0.3} \mathrm{MnO}_{3}$ thin films. Phys. Rev. B 2006, 74, 245116. [CrossRef]

35. Tebano, A.; Aruta, C.; Sanna, S.; Medaglia, P.G.; Balestrino, G.; Sidorenko, A.A.; De Renzi, R.; Ghiringhelli, G.; Braicovich, L.; Bisogni, V.; et al. Evidence of Orbital Reconstruction at Interfaces in Ultrathin $\mathrm{La}_{0.67} \mathrm{Sr}_{0.33} \mathrm{MnO}_{3}$ Films. Phys. Rev. Lett. 2008, 100, 137401. [CrossRef] [PubMed]

36. Kim, B.; Kwon, D.; Yajima, T.; Bell, C.; Hikita, Y.; Kim, B.G.; Hwang, H.Y. Reentrant insulating state in ultrathin manganite films. Appl. Phys. Lett. 2011, 99, 092513. [CrossRef]

37. Lepetit, M.B.; Mercey, B.; Simon, C. Interface effects in perovskite thin films. Phys. Rev. Lett. 2012, 108, 087202. [CrossRef] [PubMed]

38. Peng, R.; Xu, H.C.; Xia, M.; Zhao, J.F.; Xie, X.; Xu, D.F.; Xie, B.P.; Feng, D.L. Tuning the dead-layer behavior of $\mathrm{La}_{0.67} \mathrm{Sr}_{0.33} \mathrm{MnO}_{3} / \mathrm{SrTiO}_{3}$ via interfacial engineering. Appl. Phys. Lett. 2014, 104, 081606. [CrossRef]

39. Liao, Z.L.; Li, F.M.; Gao, P.; Li, L.; Guo, J.D.; Pan, X.Q.; Jin, R.; Plummer, E.W.; Zhang, J.D. Origin of the metal-insulator transition in ultrathin films of $\mathrm{La}_{2 / 3} \mathrm{Sr}_{1 / 3} \mathrm{MnO}_{3}$. Phys. Rev. B 2015, 92, 125123. [CrossRef]

40. Liao, Z.L.; Gauquelin, N.; Green, R.J.; Macke, S.; Gonnissen, J.; Thomas, S.; Zhong, Z.C.; Li, L.; Si, L.; van Aert, S.; et al. Thickness Dependent Properties in Oxide Heterostructures Driven by Structurally Induced Metal-Oxygen Hybridization Variations. Adv. Funct. Mater. 2017, 27, 1606717. [CrossRef] 
41. Porter, S.B.; Venkatesan, M.; Dunne, P.; Doudin, B.; Rode, K.; Coey, J.M.D. Magnetic Dead Layers in $\mathrm{La}_{0.7} \mathrm{Sr}_{0.3} \mathrm{MnO}_{3}$ Revisited. IEEE Trans. Magn. 2017, 53, 11. [CrossRef]

42. Moon, E.J.; Balachandran, P.V.; Kirby, B.J.; Keavney, D.J.; Sichel-Tissot, R.J.; Schlepütz, C.M.; Karapetrova, E.; Cheng, X.M.; Rondinelli, J.M.; May, S.J. Effect of Interfacial Octahedral Behavior in Ultrathin Manganite Films. Nano Lett. 2014, 14, 2509-2514. [CrossRef] [PubMed]

43. Huijben, M.; Liu, Y.H.; Boschker, H.; Lauter, V.; Egoavil, R.; Verbeeck, J.; te Velthuis, S.G.E.; Rijnders, G.; Koster, G. Enhanced Local Magnetization by Interface Engineering in Perovskite-Type Correlated Oxide Heterostructures. Adv. Mater. Interfaces 2015, 2, 1400416. [CrossRef]

44. Guo, E.J.; Roldan, M.A.; Charlton, T.; Liao, Z.L.; Zheng, Q.; Ambaye, H.; Herklotz, A.; Gai, Z.; Ward, T.Z.; Lee, H.N.; et al. Removal of the Magnetic Dead Layer by Geometric Design. Adv. Funct. Mater. 2018, 28, 1800922. [CrossRef]

45. Macke, S.; Radi, A.; Hamann-Borrero, J.E.; Verna, A.; Bluschke, M.; Brück, S.; Goering, E.; Sutarto, R.; He, F.Z.; Cristiani, G.; et al. Element Specific Monolayer Depth Profiling. Adv. Mater. 2014, 26, 6554-6559. [CrossRef] [PubMed]

46. Fister, T.T.; Fong, D.D.; Eastman, J.A.; Baldo, P.M.; Highland, M.J.; Fuoss, P.H.; Balasubramaniam, K.R.; Meador, J.C.; Salvador, P.A. In situ characterization of strontium surface segregation in epitaxial $\mathrm{La}_{0.7} \mathrm{Sr}_{0.3} \mathrm{MnO}_{3}$ thin films as a function of oxygen partial pressure. Appl. Phys. Lett. 2008, 93, 151904. [CrossRef]

47. Herger, R.; Willmott, P.R.; Schlepuetz, C.M.; Bjoerck, M.; Pauli, S.A.; Martoccia, D.; Patterson, B.D.; Kumah, D.; Clarke, R.; Yacoby, Y.; et al. Structure determination of monolayer-by-monolayer grown $\mathrm{La}_{1-x} \mathrm{Sr}_{x} \mathrm{MnO}_{3}$ thin films and the onset of magnetoresistance. Phys. Rev. B 2008, 77, 085401. [CrossRef]

48. Dulli, H.; Dowben, P.A.; Liou, S.H.; Plummer, E.W. Surface segregation and restructuring of colossal-magnetoresistant manganese perovskites, $\mathrm{La}_{0.65} \mathrm{Sr}_{0.35} \mathrm{MnO}_{3}$. Phys. Rev. B 2000, 62, R14629. [CrossRef]

49. Chen, L.N.; Wang, Z.; Wang, G.M.; Guo, H.W.; Saghayezhian, M.; Liao, Z.L.; Zhu, Y.M.; Plummer, E.W.; Zhang, J.D. Surface and Interface Properties of $\mathrm{La}_{2 / 3} \mathrm{Sr}_{1 / 3} \mathrm{MnO}_{3}$ Thin Films on $\mathrm{SrTiO}_{3}(001)$. Phys. Rev. B Condens. Matter 2008, 78, 035448. [CrossRef]

50. Kourkoutis, L.F.; Song, J.H.; Hwang, H.Y.; Muller, D.A. Microscopic origins for stabilizing room-temperature ferromagnetism in ultrathin manganite layers. Proc. Natl. Acad. Sci. USA 2010, 107, 11682-11685. [CrossRef]

51. Koohfar, S.; Disa, A.S.; Marshall, M.S.J.; Walker, F.J.; Ahn, C.H.; Kumah, D.P. Structural distortions at polar manganite interfaces. Phys. Rev. B 2017, 96, 024108. [CrossRef]

52. Pesquera, D.; Herranz, G.; Barla, A.; Pellegrin, E.; Bondino, F.; Magnano, E.; Sánchez, F.; Fontcuberta, J. Surface symmetry-breaking and strain effects on orbital occupancy in transition metal perovskite epitaxial films. Nat. Commun. 2012, 3, 1189. [CrossRef] [PubMed]

53. Sandiumenge, F.; Santiso, J.; Balcells, L.; Konstantinovic, Z.; Roqueta, J.; Pomar, A.; Espinós, J.P.; Martínez, B. Competing Misfit Relaxation Mechanisms in Epitaxial Correlated Oxides. Phys. Rev. Lett. 2013, 110, 107206. [CrossRef] [PubMed]

54. Lee, J.-S.; Arena, D.A.; Yu, P.; Nelson, C.S.; Fan, R.; Kinane, C.J.; Langridge, S.; Rossell, M.D.; Ramesh, R.; Kao, C.-C. Hidden Magnetic Configuration in Epitaxial La ${ }_{1-x} \mathrm{Sr}_{x} \mathrm{MnO}_{3}$ Films. Phys. Rev. Lett. 2010, 105, 257204. [CrossRef] [PubMed]

55. Mundy, J.A.; Hikita, Y.; Hidaka, T.; Yajima, T.; Higuchi, T.; Hwang, H.Y.; Muller, D.A.; Kourkoutis, L.F. Visualizing the interfacial evolution from charge compensation to metallic screening across the manganite metal-insulator transition. Nat. Commun. 2014, 5, 3464. [CrossRef] [PubMed]

56. Boschker, H.; Verbeeck, J.; Egoavil, R.; Bals, S.; van Tendeloo, G.; Huijben, M.; Houwman, E.P.; Koster, G.; Blank, D.H.A.; Rijnders, G. Preventing the Reconstruction of the Polar Discontinuity at Oxide Heterointerfaces. Adv. Funct. Mater. 2012, 22, 2235-2240. [CrossRef]

57. Li, L.; Liao, Z.L.; Diao, Z.Y.; Jin, R.Y.; Plummer, E.W.; Guo, J.D.; Zhang, J.D. Reentrance of low-temperature nonmetallic phase of $\mathrm{La}_{2 / 3} \mathrm{Sr}_{1 / 3} \mathrm{MnO}_{3}$ (110) thin films. Phys. Rev. Mater. 2017, 1, 034405. [CrossRef]

58. Fang, Z.; Solovyev, I.V.; Terakura, K. Phase Diagram of Tetragonal Manganites. Phys. Rev. Lett. 2000, 84, 3169. [CrossRef] [PubMed]

59. Wang, B.M.; You, L.; Ren, P.; Yin, X.M.; Peng, Y.; Xia, B.; Wang, L.; Yu, X.J.; Poh, S.M.; Yang, P.; et al. Oxygen-driven anisotropic transport in ultra-thin manganite films. Nat. Commun. 2013, 4, 2778. [CrossRef] 
60. Yang, F.; Kemik, N.; Biegalski, M.D.; Christen, H.M.; Arenholz, E.; Takamura, Y. Strain engineering to control the magnetic and magnetotransport properties of thin films. Appl. Phys. Lett. 2010, 97, 092503. [CrossRef]

61. Fang, Z.; Terakura, K. Surface Magnetic Phase Diagram of Tetragonal Manganites. J. Phys. Soc. Jpn. 2011, 70, 3356-3361. [CrossRef]

62. Koçak, A.B.; Varignon, J.; Lemal, S.; Ghosez, P.; Lepetit, M.B. Control of the orbital ordering in manganite superlattices and impact on properties. Phys. Rev. B 2017, 96, 125155. [CrossRef]

63. Liao, Z.L.; Huijben, M.; Zhong, Z.; Gauquelin, N.; Macke, S.; Green, R.J.; van Aert, S.; Verbeeck, J.; van Tendeloo, G.; Held, K.; et al. Controlled lateral anisotropy in correlated manganite heterostructures by interface-engineered oxygen octahedral coupling. Nat. Mater. 2016, 15, 425. [CrossRef] [PubMed]

64. Garcia-Munoz, J.L.; Fontcuberta, J.; Suaaidi, M.; Obradors, X. Bandwidth narrowing in bulk $\mathrm{L}_{2 / 3} \mathrm{~A}_{1 / 3} \mathrm{MnO}_{3}$. J. Phys. Condens. Matter 1996, 8, L787. [CrossRef]

65. Medarde, M.; Mesot, J.; Lacorre, P.; Rosenkranz, S.; Fischer, P.; Gobrecht, K. High-pressure neutron-diffraction study of the metallization process in $\mathrm{PrNiO}_{3}$. Phys. Rev. B 1995, 52, 9248. [CrossRef]

66. Vailionis, A.; Boschker, H.; Liao, Z.L.; Smit, J.R.A.; Rijnders, G.; Huijben, M.; Koster, G. Symmetry and lattice mismatch induced strain accommodation near and away from correlated perovskite interfaces. Appl. Phys. Lett. 2014, 105, 131906. [CrossRef]

67. Li, X.Y.; Lindfors-Vrejoiu, I.; Ziese, M.; Gloter, A.; van Aken, P.A. Impact of interfacial coupling of oxygen octahedra on ferromagnetic order in $\mathrm{La}_{0.7} \mathrm{Sr}_{0.3} \mathrm{MnO}_{3} / \mathrm{SrTiO}_{3}$ heterostructures. Sci. Rep. 2017, 7, 40068. [CrossRef] [PubMed]

68. Glazer, A.M. Classification of Tilted Octahedral in Perovskites. Acta Cryst. 1972, B28, 3384. [CrossRef]

69. Pellegrin, E.; Tjeng, L.H.; de Groot, F.M.F.; Hesper, R.; Sawatzky, G.A.; Moritomo, Y.; Tokura, Y. Soft X-ray magnetic circular dichroism study of the colossal magnetoresistance compound $\mathrm{La}_{1-x} \mathrm{Sr}_{x} \mathrm{MnO}_{3}$. J. Electron. Spectrosc. Relat. Phenom. 1997, 86, 115. [CrossRef]

70. Wadati, H.; Maniwa, A.; Chikamatsu, A.; Kumigashira, H.; Oshima, M.; Mizokawa, T.; Fujimori, A.; Sawatzky, G.A. Madelung potentials and covalency effect in strained $\mathrm{La}_{1-x} \mathrm{Sr}_{x} \mathrm{MnO}_{3}$ thin films studied by core-level photoemission spectroscopy. Phys. Rev. B 2009, 80, 125107. [CrossRef]

71. Niu, W.; Gao, M.; Wang, X.F.; Song, F.Q.; Du, J.; Wang, X.R.; Xu, Y.B.; Zhang, R. Evidence of weak localization in quantum interference effects observed in epitaxial $\mathrm{La}_{0.7} \mathrm{Sr}_{0.3} \mathrm{MnO}_{3}$ ultrathin films. Sci. Rep. 2016, 6, 26081. [CrossRef] [PubMed]

72. Lee, P.A.; Ramakrishnan, R.V. Disordered Electronic Systems. Rev. Mod. Phys. 1987, 57, 287. [CrossRef]

73. Brenig, W.; Dohler, G.H.; Heyszenau, H. Hopping conductivity in highly anisotropic systems. Philos. Mag. 1973, 27, 1093-1103. [CrossRef]

74. Feng, Y.Q.; Jin, K.J.; Gu, L.; He, X.; Ge, C.; Zhang, Q.H.; He, M.; Guo, Q.L.; Wan, Q.; He, M.; et al. Insulating phase at low temperature in ultrathin La0.8Sr0.2MnO3 films. Sci. Rep. 2016, 6, 22382. [CrossRef] [PubMed]

75. Zhan, J.M.; Li, P.G.; Liu, H.; Tao, S.L.; Ma, H.; Shen, J.Q.; Pan, M.J.; Zhang, Z.J.; Wang, S.L.; Yuan, G.L. Carrier tuning the metal-insulator transition of epitaxial $\mathrm{La}_{0.67} \mathrm{Sr}_{0.33} \mathrm{MnO}_{3}$ thin film on $\mathrm{Nb}$ doped $\mathrm{SrTiO}_{3}$ substrate. AIP Adv. 2017, 6, 045001. [CrossRef]

76. Herklotz, A.; Guo, E.J.; Wong, A.T.; Meyer, T.L.; Dai, S.; Ward, T.Z.; Lee, H.N.; Fitzsimmons, M.R. Reversible control of interfacial magnetism through ionic-liquid-assisted polarization switching. Nano Lett. 2017, 17, 1665-1669. [CrossRef] [PubMed]

77. Lu, D.; Baek, D.J.; Hong, S.S.; Kourkoutis, L.F.; Hikita, Y.; Hwang, H.Y. Synthesis of freestanding single-crystal perovskite films and heterostructures by etching of sacrificial water-soluble layers. Nat. Mater. 2016, 15, 1255. [CrossRef] [PubMed]

78. Kumah, D.P.; Disa, A.S.; Ngai, J.H.; Chen, H.H.; Malashevich, A.; Reiner, J.W.; Ismail-Beigi, S.; Walker, F.J.; Ahn, C.H. Tuning the structure of nickelates to achieve two dimensional electron conduction. Adv. Mater. 2014, 26, 1935-1940. [CrossRef]

79. Scherwitzl, R.; Gariglio, S.; Gabay, M.; Zubko, P.; Gibert, M.; Triscone, J.-M. Metal-Insulator Transition in Ultrathin $\mathrm{LaNiO}_{3}$ Films. Phys. Rev. Lett. 2011, 106, 246403. [CrossRef]

80. Groenendijk, D.J.; Autieri, C.; Girovsky, J.; Martinez-Velarte, M.C.; Manca, N.; Mattoni, G.; Monteiro, A.M.R.V.L.; Gauquelin, N.; Verbeeck, J.; Otte, A.F.; et al. Spin-Orbit Semimetal $\mathrm{SrIrO}_{3}$ in the Two-Dimensional Limit. Phys. Rev. Lett. 2017, 119, 256403. [CrossRef] 
81. Schütz, P.; Sante, D.D.; Dudy, L.; Gabel, J.; Stübinger, M.; Kamp, M.; Huang, Y.; Capone, M.; Husanu, M.-A.; Strocov, V.N.; et al. Dimensionality-Driven Metal-Insulator Transition in Spin-Orbit-Coupled $\mathrm{SrIrO}_{3}$. Phys. Rev. Lett. 2017, 119, 256404. [CrossRef] [PubMed]

82. Thomas, S.; Kuiper, B.; Hu, J.; Smit, J.; Liao, Z.L.; Zhong, Z.; Rijnders, G.; Vailionis, A.; Wu, R.; Koster, G.; et al. Localized Control of Curie Temperature in Perovskite Oxide Film by Capping-Layer-Induced Octahedral Distortion. Phys. Rev. Lett. 2017, 119, 177203. [CrossRef] [PubMed] 